\title{
TRADUÇÃo DO TEXTO “RÉFLEXIONS ET ECLAIRCISSEMENS SUR LES NOUVElles Vibrations des CORDES EXPOSÉES DANS LES MÉMOIRES DE L'ACADÉMIE DE 1747 \& 1748”, DE DANIEL BERNOULLI
}

\author{
Oscar João Abdounur \\ Universidade de São Paulo - USP - Brasil \\ Glauco Aparecido de Campos \\ Instituto Federal de São Paulo - IFSP - Brasil \\ (aceito para publicação em setembro de 2021)
}

\begin{abstract}
Resumo
Este artigo apresenta uma tradução do texto Réflexions et Eclaircissemens sur les Nouvelles Vibrations des Cordes Exposées dans les Mémoires de l'Académie de 1747 \& 1748 do estudioso Daniel Bernoulli (1700-1782), publicado nas Mémoires de l'Académie Royale des Sciences et Belles-Lettres em Berlim. Este texto fora escrito em resposta às memórias De vibratione chordarum exercitatio (1748) de Leonhard Euler (1707-1783) e Recherches sur la courbe que forme une corde tendue, mise en vibration (1747), de Jean le Rond d'Alembert (1717-1783), trabalhos que também foram publicados nas Mémoires da academia mencionada. Em contrapartida ao tratamento matemático apresentado por Euler e d’Alembert, Bernoulli procura construir uma justificativa para a percepção dos sons ouvidos simultaneamente ao som principal de uma corda vibrante, por meio da sobreposição dos modos de vibração de uma corda qualquer, dando continuidade a uma acirrada disputa sobre a questão. Em tal abordagem, é fundamental ressaltar que Bernoulli mantém continuamente um sentido físico para as teorizações dos sons simultâneos por ele estabelecidos e inferências delas decorrentes.
\end{abstract}

Palavras-chave: Cordas vibrantes, abordagem física, harmônicos, história da acústica 


\title{
TRANSLATION OF THE TEXT "RÉFLEXIONS ET ECLAIRCISSEMENS SUR LES NOUVElles Vibrations des CoRdes EXPOSÉES DANS LES MÉMOIRES DE L'ACADÉMIE DE 1747 \& 1748”, BY DANIEL BERNOULLI
}

\begin{abstract}
This article presents a translation of the text Réflexions et Eclaircissemens sur les Nouvelles Vibrations des Cordes Exposées dans les Mémoires de l'Académie de 1747 \& 1748 written by the scholar Daniel Bernoulli (1700-1782), published in Mémoires de l'Académie Royale des Sciences et Belles-Lettres in Berlin. It was written as an answer to the memoirs De vibratione chordarum exercitatio (1748) de Leonhard Euler (1707-1783) and Recherches sur la courbe que forme une corde tendue, mise en vibration (1747), de Jean le Rond d'Alembert (1717-1783), two works which were also published in the Mémoires of the academy mentioned above. In contrast to the mathematical approach presented by Euler and d'Alembert, Bernoulli seeks to build a justification to the perception of the sounds listened simultaneously with the main sound of a vibrating string, via superposition of vibration modes of an arbitrary string, carrying on to a tough dispute about the issue. In this approach, it is fundamental to point out that Bernoulli mantains a physical meaning to the theorizations of the simultaneous sounds established by him and inferences resulted by them.
\end{abstract}

Keywords: Vibration strings, physical approach, overtones, history of acoustic.

\section{Apresentação}

Uma evidência representativa da abordagem física referente à percepção dos sons percebidos simultanemente se dá quando Euler critica argumentos físicos de Bernoulli, por exemplo, opondo-se ao fato de que o enésimo harmônico de uma corda deveria ser uma senoide $^{1}$, o que Euler considerava uma particularização. Fundamentado em Taylor, Bernoulli concebia um harmônico como uma senoide (companheira de uma cicloide extremamente alongada), ainda por que ele não compreendia em que sentido outras curvas poderiam ser admitidas. A abordagem citada é talvez a razão pela qual Bernoulli vê a necessidade de responder aos textos dos estudiosos mencionados.

As tentativas de Bernoulli de racionalizar os harmônicos² tendo por base a corda vibrante parece permitir a ele a possibilidade de conceber tais sons para qualquer corpo sonoro, que, portanto, possui uma infinidade de sons e uma infinidade de maneiras correspondentes de fazer vibrações regulares. O tipo de inferência mencionado (analogia) é bastante recorrente na argumentação utilizada por Bernoulli no presente texto.

\footnotetext{
${ }^{1}$ DARRIGOL, 2007, p. 361.

${ }^{2}$ Na realidade, Bernoulli não utilizava este termo, mas optamos por utilizá-lo nesta introdução para a facilitar a compreensão.
} 
Bernoulli trata a composição de sons distintos de maneira semelhante com que trata a composição de harmônicos de um som, estabelecendo uma analogia entre a percepção de fontes sonoras distintas em um concerto com o quanto os respectivos harmônicos de tais fontes não se impedem. Com isso, Bernoulli está concebendo o que a historiografia costuma chamar de princípio da superposição.

Do ponto de vista estilístico, Bernoulli apresenta seu texto muitas vezes com frases mais espontâneas, o que por um lado dificulta a apreensão do encadeamento lógico subjacente ao texto, mas por outro favorece a compreensão da natureza heurística do pensamento deste estudioso do século XVIII. Na presente tradução, optamos por respeitar ao máximo a estilística/estrutura do texto, fazendo as mínimas mudanças suficientes para dar sentido ao texto em português.

\section{Bibliografia}

BERNOULLI, Daniel. 1753. Réfléxions et Eclaircissemens sur les Nouvelles Vibrations des Cordes Exposées dans les Mémoires de l’Academie de 1747 \& 1748. In: Mémoires de l'Academie Royale des Sciences et Belles-Lettres, tom. IX. 147-172.

DARRIGOL, Olivier. 2007. The acoustic origins of harmonic analysis. In: Archive for history of exact sciences, vol. 61, n 4. 343-424.

TRUESDELL, Clifford. 1960. The rational mechanics of flexible or elastic bodies 16381788. In: Leonhardi Euleri Opera Omnia, vols. X e XI, Ser. 2.

\section{Oscar João Abdounur}

Departamento de Matemática - Mat - campus São

Paulo- Brasil

E-mail: abdounur@ime.usp.br

\section{Glauco Aparecido de Campos}

Instituto Federal de São Paulo - IFSP - campus

Bragança Paulista - Brasil

E-mail: glaucodecampos@ifsp.edu.br 


\title{
Reflexões e esclarecimentos sobre as novas vibrações de cordas expostas nas memórias da Academia de 1747 e 1748
}

\author{
por Daniel Bernoulli
}

\section{Introdução}

I. O sr. Taylor veio a ser o primeiro a conhecer o número de vibrações, que faz em um tempo dado uma corda uniformemente espessa com um comprimento dado, com um peso dado e tensionada por uma força dada. Não seria possível determinar esse número sem conhecer previamente as curvas, que formariam as cordas durante todo o tempo que durariam suas vibrações; foi, portanto, demonstrado, que essa curva seria constantemente a companheira de uma cicloide extremamente alongada, sobre a qual as ordenadas representam o seno dos arcos representados pelas abscissas: também é a meu ver que sob esta forma as vibrações podem se tornar regulares, simples e isócronas, apesar da desigualdade de excursões. Com esta ideia que eu sempre tive, eu só posso estar surpreso de ver nos Mémoires dos anos 1747 e 1748 uma infinidade de outras curvas dotadas da mesma propriedade; não me seria necessário menos que os grandes nomes dos srs. d'Alembert e Euler, que eu não posso suspeitar de qualquer desatenção, para examinar se não existiria algum equívoco na agregação de todas estas curvas com aquela do sr. Taylor, e em qual sentido poder-se-ia admiti-las. Eu vi imediatamente, que só se pode admitir esta multitude de curvas num sentido absolutamente impróprio; eu não estimo menos os cálculos dos srs. d'Alembert e Euler, que abarcam certamente tudo que a análise pode ter de mais profundo e mais sublime; mas que mostram ao mesmo tempo, que uma análise abstrata, que se leva em consideração sem qualquer exame sintético da questão proposta, está sujeita a nos surpreender mais do que nos esclarecer. Parece-me que bastaria dar atenção à natureza de vibrações simples das cordas, para prever sem quaisquer cálculos tudo que estes dois grandes geômetras encontraram pelos cálculos mais espinhosos e mais abstratos, com um espírito analítico que permanecesse atento.

II. Notemos inicialmente que, segundo a teoria do sr. Taylor, uma corda tensionada pode formar suas vibrações uniformes de uma infinidade de maneiras, que diferem entre elas para a física, mas que retornam às mesmas na geometria, uma vez que em cada uma dessas maneiras faz-se somente mudar a unidade que serve de medida. Estas diferentes maneiras consistem no número de ventres, que a corda pode formar durante suas vibrações. Quando existe somente um ventre, como na primeira figura ${ }^{3}$, as vibrações são mais lentas, elas formam o som fundamental; quando existem dois ventres, e um nó no meio do eixo, as vibrações dobram, e elas formam a oitava do som fundamental; quando a corda forma três, quatro ou cinco ventres, com dois, três ou quatro nós, segundo distâncias mútuas iguais, como nas figuras 3,4 e 5 , as vibrações triplicam, quadruplicam, ou

${ }^{3}$ As figuras se encontram na última página do original francês, após o fim da tradução. 
quintuplicam, e formam a décima-segunda, a oitava dupla, ou a terça maior da oitava dupla, relativamente ao som fundamental. Esta multiplicidade vai ao infinito. Em cada espécie ${ }^{4}$ destas vibrações, as excursões totais podem ser grandes ou pequenas a critério, dado que as maiores podem ser consideradas extremamente pequenas. A natureza destas vibrações é tal que não somente cada ponto começa e termina cada vibração simples ao mesmo tempo, mas ainda que todos os pontos se colocam após cada meia vibração simples na posição do eixo $A B$. É necessário considerar todas estas condições como essenciais, e disso decorre imediatamente que há apenas as curvas dadas por Taylor que satisfazem o problema. Mas ao separar estas condições pode-se formar uma infinidade de curvas, que satisfazem alguma condição separadamente; mas eu mostrarei quão pouco seria fundamentado nestes casos recorrer às vibrações isócronas para cada ponto. Estas vibrações são como movimentos recíprocos dos corpos, que descem e sobem alternadamente sobre uma curva; esta curva só pode ser a cicloide, se for requerido que todas as descidas, grandes ou pequenas, sejam isócronas entre elas da mesma forma que as subidas; mas se se quer simplesmente que as vibrações inteiras sejam isócronas entre elas, se pode dar tantas curvas quanto se queira, que satisfazem a este problema; uma vez que eu demonstrei nos Mémoire de Petersburgo, que para qualquer que seja a curva de descida dada, pode-se sempre determinar a curva de subida, tal que os dois tempos empregados para a descida e para a subida tomados juntamente resultem numa mesma soma, qualquer que seja a desigualdade existente entre as amplitudes das excursões.

III. Se eu disse que as cordas podem fazer suas vibrações simples de uma infinidade de maneiras, em que as cinco primeiras figuras servem de exemplo, isto não é somente uma verdade abstrata; pode-se fazê-la real, como o exemplo dos trompetes marinhos o mostra suficientemente pelos sons que se tira: as experiências feitas ao se colocar um cavalete em algum dos pontos $a$, e ao pinçar o pedaço $A a$ confirmam a mesma coisa; uma vez que elas [as experiências] nos ensinam que se formam nos pontos $b, c$ e $d$ os nós a distâncias iguais, que permanecem em repouso, enquanto todos os outros pontos estão em movimento. Esta multiplicidade infinita de vibrações manifesta-se em todos os corpos sonoros, independentemente da natureza que eles possam ter; é por isso que se pode tirar da trompa, do trompete etc. todos os sons que vão em progressão de números naturais; quer dizer, na mesma progressão dos sons, que se pode tirar de uma única e mesma corda pelas diferentes espécies de vibrações. Ao fechar-se todos os buracos de uma flauta transversal, pode-se pela simples variação de embocadura obter primeiramente o som mais grave ou fundamental, e depois sucessivamente sua oitava, sua décima-segunda, sua oitava dupla, sua décima-sétima maior, que semelhantemente são como 1, 2, 3, 4 e 5, mas não é necessário crer que esta progressão seja geral. Após ter formado uma boa teoria sobre as vibrações de ar nos instrumentos de sopro, eu concluí que que só se poderia tirar de tubos fechados os sons que vão em progressão de números ímpares, a saber, 1, 3, 5, 7 etc., e minha conclusão foi confirmada pela experiência: pois tendo retirado a peça superior de uma flauta transversal e a fechando com a mão, eu tirei primeiramente o som mais grave, e depois reforçando o sopro sua décima-segunda sem

${ }^{4}$ Em relação ao termo espécie, podemos entendê-lo como modo de vibração de uma corda. 
passar para a oitava, em seguida a décima-sétima maior, e enfim um tom que não é reconhecido na música, e que se aproximava da vigésima-primeira do som fundamental. Eu não sei se esta propriedade já foi observada por outros; mas ela me parece ainda mais notável, pois talvez seja adequada para explicar em que consistem esses acessos de fácil reflexão e de fácil transmissão dos raios, observados com tanta sagacidade pelo grande Newton. É sobre isto que eu considero fazer um Mémoire, na qual eu teria explicado e reduzido [a propriedade] ao cálculo de vibrações de ar formadas nos tubos abertos e fechados, e demonstrado a analogia entre estas vibrações e aquelas do éter, que faz a luz. Eu faço aqui esta observação para provar, que os diferentes sons tirados de um mesmo corpo sonoro não vão sempre na progressão dos números naturais. Mas eu digo mais: estes sons podem ter tal proporção a ponto de não existir qualquer fórmula em quantidade finita que possa exprimi-la; como se pode ver pelos sons, que eu calculei uma outra vez, a saber, aqueles que se podem tirar de uma vara de aço batidas pelos pequenos golpes. Era um problema novo, que demandava muito de circunspecção. Depois de tê-lo resolvido, eu o propus ao sr. Euler, que o deu uma solução perfeitamente conforme a minha, ainda que em princípio incompleta, na medida em que ele tinha omitido a metade dos sons possíveis. Eu o adverti e ele a completou nas atas de Leipzig.

IV. Minha conclusão é, que todos os corpos sonoros contêm em potencial uma infinidade de sons, e uma infinidade de maneiras correspondentes de fazer suas vibrações regulares; enfim, que em cada espécie diferente de vibrações as inflexões das partes do corpo sonoro se fazem de uma maneira diferente. Mas não é dessa multitude de vibrações aplicada a cordas tensas, que os srs. d’Alembert e Euler pretendem falar; ela não era desconhecida ao sr. Taylor: eles multiplicam ao infinito cada espécie [eles argumentam que existem infinitas espécies]; em concordância com cada intervalo entre dois nós vizinhos [existe] uma infinidade de curvas tais que cada ponto começa e conclui ao mesmo instante as vibrações, enquanto que, seguindo a teoria de Taylor, cada dito intervalo deve necessariamente tomar a única curva da companhia da cicloide extremamente alongada [senoide]. Esta contradição aparente entre os também grandes geômetras me parece demandar algum esclarecimento.

V. Observemos, portanto, que a corda $A B$ pode não somente fazer suas vibrações de acordo com a primeira figura, ou a segunda, ou a terceira e assim por diante, mas podese fazer ainda uma mistura de todas estas vibrações com todas as combinações possíveis; e, portanto, todas as novas curvas e novas espécies de vibrações dadas pelos srs. d'Alembert e Euler são apenas uma mistura de várias espécies de vibrações taylorianas. Se isto é verdade, eu não saberia aprovar a agregação de todas estas curvas novas; uma vez que a corda, ao se adequar a ela não dá um único tom, mas vários tons ao mesmo tempo. Para perceber a incongruência dessa nova agregação, suponhamos que, no lugar de uma só corda, [tenhamos] por exemplo cinco cordas inteiramente iguais em todos os sentidos, e que a primeira faça as suas vibrações de acordo com a lei da primeira figura; a segunda corda de acordo com a lei da segunda figura, e assim por diante. Supondo que todas estas cordas comecem suas vibrações em um mesmo instante; querer-se-ia chamar estas cinco espécies de vibrações de isócronas entre elas, simplesmente porque elas terminam uma de suas 
vibrações ao mesmo tempo que a primeira corda termina cada uma das suas? Sem dúvida, uma vez que a segunda corda faz duas vibrações, a terceira três, e assim por diante, enquanto que a primeira corda só faz uma; e que cada corda dá um tom diferente em proporção. Entretanto, tudo que eu acabei de dizer com relação a várias cordas inteiramente iguais, pode e deve ser aplicado a uma única corda.

VI. Efetivamente, todos os músicos concordam, que uma corda longa tocada dá ao mesmo tempo, além de seu tom fundamental, outros tons bem mais agudos; eles observam sobretudo a mistura da décima-segunda e da décima-sétima maior: eles também não observam distintamente a oitava da oitava dupla, isto é somente por causa da bem grande semelhança destes dois tons com o tom fundamental. Aqui está uma prova evidente de que se pode fazer em uma única corda uma mistura de vários tipos de vibrações taylorianas de uma vez. Ouve-se de forma semelhante nos sons de grandes sinos uma mistura de tons diferentes. Se se segurar na metade um bastão de aço, no qual se bate, ouve-se de uma vez uma mistura confusa de vários tons, os quais, sendo apreciados por um músico hábil, encontram-se extremamente desarmoniosos, de maneira que se forma aqui uma concorrência de vibrações, que nunca começam e terminam em um mesmo instante, senão por um grande acaso: de onde se pode ver que a harmonia de sons, que se ouve em um mesmo corpo sonoro de uma vez, não é essencial a esta matéria [harmonia], e não deve servir de princípio para os sistemas de música. $\mathrm{O}$ ar não está isento desta multiplicidade de sons coexistentes: ocorre frequentemente que se tira de uma vez dois sons diferentes de um tubo; mas, aquilo que se prova melhor, quão pouco as diferentes ondulações de ar se perturbam, é quanto se ouve distintamente todas as partes de um concerto, e que todas as ondulações causadas por estas diferentes partes formam-se em uma mesma massa de ar sem se perturbar mutuamente, tudo como os raios da luz, que entram em um quarto obscuro através de uma pequena abertura, não se perturbando.

VII. Depois destas observações, será fácil construir uma infinidade de curvas a partir da curva $A B$ com a condição de que cada ponto da corda chegue alguma vez ao mesmo tempo a um ponto de repouso instantâneo, e dar a lei geral para todas estas curvas sem qualquer cálculo preliminar. Comecemos pela combinação das duas primeiras figuras. Suponhamos que a corda faça suas vibrações para formar o som fundamental conforme a primeira figura; esta curva sendo supostamente infinitamente pequena, a corda poderia ainda ser considerada como uma linha reta, e sua curva poderia servir de eixo móvel à curva da segunda figura, a dois ramos; e disso resulta uma nova curva, que preencheria a condição prescrita. Aqui está, portanto, a construção desta nova curva.

VIII. Seja (fig. 6) $A \operatorname{man} B$ a curva da primeira figura: considera-se esta curva como um eixo reto, sobre o qual se constrói $A p a q B$ inteiramente a mesma com relação ao seu eixo curvo que aquela da figura segunda, com relação ao eixo perfeitamente reto $A B$, e esta curva $A p a q B$ será tal qual se desejava, a mais simples das curvas dadas pelos srs. d'Alembert e Euler. 
Como a aplicada [ordenada] maior $p m$ pode ter uma certa razão em relação à aplicada [ordenada] maior $a r$, está claro que esta curva $A p a q B$ já contém uma infinidade de espécies. Eis aqui as propriedades desta curva $A p a q B$ :

(a) Eu digo que a curva ideal $A \operatorname{man} B$ fará suas vibrações com relação ao eixo reto $A r B$ seguindo inteiramente a lei de vibrações simples da primeira figura.

(b) Em seguida, que cada ponto da curva $A p a q B$ terá seu movimento relativo com relação a cada ponto correspondente da curva $\operatorname{Aman} B$, o mesmo que o movimento absoluto representado pela segunda figura.

(c) No entanto, cada ponto da segunda figura faz precisamente duas vibrações, enquanto o mesmo ponto faz uma nas vibrações da primeira figura. É necessário portanto que todos os pontos da curva $A p a q B$ terminem suas vibrações alternadas ao mesmo tempo, que aquelas da figura $A \operatorname{man} B$ terminam cada uma de suas.

(d) No início, uma vez que um ponto da curva $A p a q B$ está fora da curva $A \operatorname{man} B$, este mesmo ponto no começo de suas duas vibrações estará dentro desta última curva, que não terá feito uma única vibração, e assim a curva $A \operatorname{man} B$ tendo assumido a situação $A m^{\prime} a^{\prime} n^{\prime} B$, acontecerá que a curva $A p a q B$ assuma a situação $A p^{\prime} a^{\prime} q^{\prime} B$, e que a curva $A p^{\prime} a^{\prime} q^{\prime} B$ seja completamente a mesma que ela tinha sido, mudando a ordem dos lados, quer dizer, mesma que $B q a p A$, o que faz um caso do belo teorema do sr. Euler.

IX. Da mesma forma que nós combinamos as vibrações da primeira e da segunda figura, nós poderemos combinar as vibrações da primeira figura com aquelas de todas as outras figuras sem exceção até o infinito; todas estas combinações podem subsistir ao mesmo tempo; assim; por exemplo, a curva absoluta $A p a q B$ da sexta figura, poderá ser ainda considerada como uma curva transladada, poder-se-á reparti-la em três partes iguais, e aplicar aí a mesma curva que, na terceira figura, é aplicada ao eixo reto $A B$. Qualquer que seja a curva absoluta resultante de todas estas combinações feitas a critério, ocorrerá sempre que todos os pontos da curva chegam num mesmo instante ao ponto de repouso instantâneo do lado oposto do eixo, e a curva neste estado será sempre a mesma, em situação oposta, como ela foi no começo, como o diz o sr. Euler.

$\mathbf{X}$. Se se combina as figuras que têm um número de ventres ímpares, estas curvas ainda terão a propriedade de que cada ponto da curva absoluta passe num mesmo instante pelo eixo reto $A B$, o que se pode fazer consequentemente de uma infinidade de maneiras, mas assim que uma só curva com ventres pares se mistura, isso não acontece mais. Veja lá uma exposição física das novas vibrações de cordas dadas pelos srs. d'Alembert e Euler, e se eu compreendi bem seus enunciados, todas as curvas novas que eles dão, estão incluídas na nossa construção, e são uma simples mistura de várias espécies de vibrações, em que cada uma tomada a parte segue as leis descritas pelo sr. Taylor. Mas me parece que isto só é 
uma espécie de composição de movimento, que não pode dar qualquer amplificação à teoria do sr. Taylor.

XI. Para melhor compreender a incongruência de uma tal amplificação, nós combinaremos a curva fundamental do sr. Taylor, que é representada pela primeira figura, com a curva anguiforme tayloriana que teria 1001 ventres; resultará uma curva [composta] de número de novas curvas, que terá a propriedade de que todos os pontos começam ao mesmo tempo suas vibrações, passarão todos num mesmo instante pelo eixo reto, e chegarão em um mesmo instante ao ponto de repouso, do outro lado do eixo, para começar um novo movimento semelhante ao precedente. Mas o que ocorre durante este movimento? Eu digo que haverá na corda precisamente 1000 pontos a distâncias pequenas iguais, em que cada um faz uma vibração absolutamente da mesma maneira como se as vibrações se fizessem simplesmente seguindo a lei da primeira figura, enquanto que todos os outros pontos farão 1001 idas e vindas, ou 1001 vibrações, e poderão mesmo passar 1001 vezes o eixo reto. Estas 1001 vibrações serão inteiras e perfeitas; em cada dessas vibrações cada elemento terá um momento de repouso absoluto e perfeito, e um momento no qual sua velocidade seja a maior. Se desejássemos confundir essas pequenas vibrações rápidas com a vibração simples fundamental, unicamente porque a milésima primeira vibração pequena termina ao mesmo tempo em cada ponto, que a vibração fundamental termina, isto seria chamar síncronos dois pêndulos simples, em que um teria um pé de comprimento e o outro 10020001 pés, pois estes dois pêndulos começavam suas vibrações ao mesmo tempo, as terminam também ao mesmo tempo; se poderia mesmo unir estes dois pêndulos, em supondo um corpo extremamente pesado, suspenso por uma corda longa, e um pequeno corpo anexado ao grande por um pequeno fio, poder-se-á sempre obter que o pequeno pêndulo anexado ao grande começa a primeira de suas vibrações e termina a última, ao mesmo tempo que o grande pêndulo começa e termina uma única vibração; mas será que haverá fundamento para chamar estas vibrações de isócronas?

XII. Vejamos ainda se todas as novas curvas encontradas pelo sr. Euler são compreendidas na nossa observação. Para este efeito, será necessário dar uma equação para todas as curvas taylorianas, das quais as cinco primeiras figuras são exemplos. Eu me servirei das denominações do sr. Euler. Seja, portanto, o comprimento da corda $A B=a$; $\pi=$ a semi-circunferência do círculo cujo raio é expresso pela unidade, a maior ordenada no meio de cada ventre para a primeira figura $\alpha$, para a segunda $\beta$, para a terceira $\gamma$, para a quarta $\delta$, seja enfim $x$ uma certa abscissa, e $y$ a ordenada para essa abscissa, se terá segundo o sr. Taylor.

$$
\begin{aligned}
& \text { para a } 1^{\text {a }} \text { figura } y=\alpha \operatorname{sen} \frac{\pi x}{a} \\
& \text { para a } 2^{\mathrm{a}} \text { figura } y=\beta \operatorname{sen} \frac{2 \pi x}{a}
\end{aligned}
$$




$$
\begin{aligned}
& \text { para a } 3^{\text {a }} \text { figura } y=\gamma \operatorname{sen} \frac{3 \pi x}{a}
\end{aligned}
$$

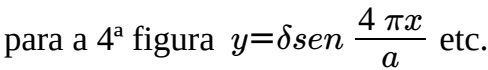

Portanto, combinando todas estas curvas à imitação da sexta figura, para a qual nós combinamos as duas primeiras figuras, nós teremos geralmente para a mesma abscissa $x$ esta equação:

$$
y=\alpha \operatorname{sen} \frac{\pi x}{a}+\beta \operatorname{sen} \frac{2 \pi x}{a}+\gamma \operatorname{sen} \frac{3 \pi x}{a}+\delta \operatorname{sen} \frac{4 \pi x}{a} \text { etc. }
$$

na qual as quantidades $\alpha, \beta, \gamma, \delta$ etc são arbitrariamente positivas ou negativas.

XIII. Veja lá esta infinidade de curvas encontradas sem qualquer cálculo, e nossa equação é a mesma que aquela do sr. Euler; vide as Mémoires de l'Academie para o ano 1748, página 85. É verdade que o sr. Euler, não trata esta multitude infinitamente infinita [infinita] em geral, e que ele somente a dá no §. 30 como casos particulares; mas é sobre isto que eu não estou suficientemente esclarecido: se existe ainda outras curvas, eu não compreendo em que sentido se pode as admitir.

XIV. Se na nossa equação, se supuser os coeficientes dos termos alternados, a saber $\beta, \delta=0$, ocorrerá, portanto, não somente, que todas as espécies de vibrações terminam ao mesmo tempo que termina a vibração fundamental, mas ainda que todos os pontos da corda se organizam ao mesmo tempo na posição da linha reta $A B$; isto não é senão um corolário do nosso §.10. Nesta suposição, a curva obtém um diâmetro que passa pela metade da corda $A B$ e que reparte a curva em dois ramos iguais e semelhantes. Este teorema é, portanto, ainda o mesmo que aquele do sr. Euler do §. 28.

XV. Se após todas as nossas observações querer-se-ia ainda confrontar as vibrações compostas com as vibrações simples expostas pelo sr. Taylor, eu não me oporia; minha intenção não foi senão principalmente a de expor aquilo que as novas vibrações dos srs. d’Alembert e Euler tem de físico. Se ao contrário, encontra-se que estas novas vibrações não saberiam ser consideradas como vibrações simples, que somente faziam o objeto do sr. Taylor, e que estando decompostas em vibrações simples e uniformes, cada espécie se faz simplesmente seguindo a lei de Taylor, estas novas curvas só farão confirmar a teoria de Taylor, quando ele exclui todas as outras curvas e ele admite somente a trocoide prolongada. Mas eu não admiraria menos a profunda sagacidade com a qual nossos dois ilustres geômetras souberam determinar analiticamente estas novas curvas. De resto, eu creio que qualquer curva inicial que se dê à corda, ela não se furtará de fazer suas vibrações quase que imediatamente seguindo a simples uniformidade de movimentos isócronos, e em conformidade com a natureza da trocoide prolongada exposta pelo sr. Taylor, embora o sr. Euler não pareça [compartilhar] deste sentimento no §. 27. Aqui está como eu concebo a 
coisa. A experiência e a razão nos ensinam, que de duas cordas igualmente grandes e igualmente tensionadas, a mais longa conserva suas vibrações por mais tempo do que a menor, nas cordas pequenas tocadas, o som dura somente um instante. Assim todas as vibrações parciais, misturadas com a vibração total e fundamental, terminarão bem rapidamente, enquanto que a fundamental dura ainda bem sensivelmente, e, portanto, a corda não formará mais do que a trocoide do sr. Taylor. É também isto que nós vemos sempre ocorrer nas cordas pinçadas de um cravo, nas quais se reconhece pelos olhos tal curva uniforme, que só forma um único ventre.

XVI. Pode-se ainda observar acerca desta matéria, que há somente cordas uniformemente espessas suscetíveis de propriedades que nós acabamos de expor; a razão é, que quando as cordas irregularmente espessas se dobram segundo a figura 2, 3, 4, 5 etc. as vibrações não se tornam precisamente 2, 3, 4, 5 etc. vezes mais rápidas que elas são com respeito à primeira figura, e que assim na mistura destas vibrações elas não terminam nunca em um mesmo instante, embora elas tenham todas começado em um mesmo instante. Não existe, portanto, a meu ver uma única curva para as cordas desigualmente espessas, para um número de intersecções dadas ao nosso problema; e se é assim, por que existiria uma infinidade para as cordas uniformemente espessas? Enfim, que se escolha a mistura mais simples, considerando a equação $y=\alpha \operatorname{sen} \frac{\pi x}{a}+\beta \operatorname{sen} \frac{2 \pi x}{a}$, na qual os coeficientes $\alpha$ e $\beta$ podem ser certas linhas pequenas. Seja o comprimento do pêndulo simples isócrono, com as vibrações uniformes que correspondem à primeira figura (simples) $l$; 0 comprimento de um pêndulo semelhante para a segunda figura $\frac{1}{4} l$; ou segundo os srs. d'Alembert e Euler, o pêndulo isócrono para a figura expressa pela equação $y=\alpha \operatorname{sen} \frac{\pi x}{a}+\beta \operatorname{sen} \frac{2 \pi x}{a}$ tem sempre para comprimento a quantidade $l$; embora este mesmo comprimento é manifestamente $\frac{1}{4} l$ fazendo $\alpha=0$; existiria então aí uma contradição, se estes geômetras não tomassem a palavra isocronismo num outro sentido, que não aquele vinculado a ele ordinariamente.

XVII. Da nossa solução sintética do problema dos srs. d'Alembert e Euler, se vê também sem cálculo a maneira de se determinar a curva absoluta da corda a cada instante, da mesma forma a velocidade de cada ponto: pois, seja a curva inicial expressa por:

$$
y=\alpha \operatorname{sen} \frac{\pi x}{a}+\beta \operatorname{sen} \frac{2 \pi x}{a}+\gamma \operatorname{sen} \frac{3 \pi x}{a}+\delta \operatorname{sen} \frac{4 \pi x}{a}+e t c .
$$

e seja, por exemplo, a questão de determinar a curva no momento em que o meio da corda passa pelo eixo reto $A B$ : é claro que a curva será expressa neste instante pela relação:

$$
y=-\beta \operatorname{sen} \frac{2 \pi x}{a}+\delta \operatorname{sen} \frac{4 \pi x}{a}-e t c .
$$


na qual os sinais são alternadamente negativos e positivos. Quanto às velocidades, é igualmente fácil determiná-las pela simples composição de movimento, uma vez que se determina na mecânica a razão de velocidades de cada ponto para cada figura à parte.

XVIII. Eu evitei até aqui os cálculos, e eu fundamentei todo o meu raciocínio sobre o princípio confirmado pela experiência (§. 6.), que pode se fazer uma mistura de vibrações em um único e mesmo corpo sonoro, que sejam absolutamente independentes umas das outras. Considerando cuidadosamente este princípio, ele não é diferente daquele da composição de movimento, entretanto, para atualizá-lo, eu acreditei juntar aqui as reflexões mecânicas, e os cálculos que esta matéria demanda.

XIX. Seja um corpo $A$ (fig. 7) arrastado diretamente em direção a um ponto fixo $B$; se se exige que o corpo chegue ao ponto $B$ em um tempo dado, qualquer que seja a distância inicial $A B$, tem-se que as atrações do corpo $A$ em direção ao ponto fixo $B$ devem ser em cada ponto proporcionais às distâncias dos corpos desde o ponto $B$. Tem-se mesmo que existe somente esta lei da gravitação que satisfaça à questão, então as meias excursões se tornam de uma parte e de outra perfeitamente iguais. Aí está o verdadeiro isocronismo e o único que deve ser considerado. Suponhamos após isto que, enquanto o corpo $A$ é empurrado com a dita lei em direção ao ponto $B$, os pontos $A$ e $B$ sofrem em cada instante uma aceleração igual em direção ao ponto $C$, de modo que o sistema $A B$ sofre uma gravitação comum em direção a $C$, que seja ainda proporcional às distâncias $B C$, deste modo obter-se-á um outro isocronismo nas excursões do sistema $A B$. Após isso, poder-se-á novamente imaginar que os três pontos $A, B$ e $C$, sofrem em cada instante uma aceleração igual em direção a um quarto ponto $D$ e que esta aceleração comum aos três pontos $A, B$ e $C$ seja sempre proporcional às distâncias do ponto $C$ ao ponto $D$. Desta maneira, poder-se-á multiplicar ao infinito as gravitações do corpo $A$ em direção a diferentes pontos, e esse corpo $A$ sofrerá desse modo uma mistura de várias espécies de vibrações, que convém examinar.

XX. Qualquer que seja a distância inicial, o corpo $A$ empregará sempre o mesmo tempo para alcançar o ponto $B$, e para fazer uma semi-vibração; após o que o corpo $A$ fará uma outra semi-vibração, do lado oposto sempre no mesmo momento; ele retornará ainda ao ponto $B$ após um terceiro momento, e assim por diante: em uma palavra, esse movimento relativo ao ponto $B$ fará o mesmo, que se este ponto permanecesse inteiramente em repouso. Eu chamaria estes movimentos recíprocos de vibrações isócronas relativas; e essa distinção das vibrações é bem necessária, pois de fato eles são bem diferentes das vibrações absolutas do mesmo corpo $A$. Suponhamos que o peso do corpo $A$ em direção ao ponto $B$ seja igual ao peso natural, fazendo a distância do ponto $B=a$; nessa suposição estas primeiras vibrações isócronas relativas serão isócronas com as vibrações absolutas de um pêndulo simples de comprimento $a$.

Após ter considerado estas primeiras vibrações relativas ao ponto $B$, nós teremos as mesmas reflexões a fazer sobre as vibrações isócronas do ponto $B$ relativas ao ponto $C$ ; e estas vibrações existirão ao mesmo tempo no corpo $A$, sem perturbar de qualquer maneira as primeiras vibrações desse corpo. Estas segundas vibrações serão isócronas com 
aquelas de um pêndulo simples de comprimento $b$, supondo que o peso do ponto $B$, ou do corpo $A$, em direção ao ponto $C$, se torna igual ao peso natural, onde se faz $B C=b$.

Se se quer juntar a estas duplas vibrações do corpo $A$ uma terceira espécie, será necessário supor uma terceira vibração comum ao sistema precedente $A B C$ em direção ao ponto $D$, que estará sob denominações isócronas semelhantes com aquelas de um pêndulo simples de comprimento $c$. Desta maneira se poderá ir tão longe quanto se queira e produzir no corpo $A$ tantas espécies de vibrações isócronas relativas quanto se será proposto. Mas não vamos mais longe, e suponhamos esta terceira vibração absoluta, considerando o ponto $D$ como fixo. Desta maneira o único e mesmo corpo $A$ terá ao mesmo tempo três espécies de vibrações independentes umas das outras, cujos centros de força são os pontos $B, C$, e $D$, que nós vamos considerar mais de perto.

XXI. Os tempos das referidas vibrações parciais estarão sob razão dupla [quadrada] das quantidades arbitrárias $a, b$ e $c$; se estas quantidades são como 4, 9 e 36, os tempos das vibrações serão como 2, 3 e 6; assim, enquanto o ponto $C$ faz uma vibração inteira relativamente ao ponto fixo $D$, o ponto $B$ fará duas relativamente ao ponto $C$, e o ponto $A$ fará três relativamente ao ponto $B$ : de onde é claro, que todo o sistema não será retornado ao seu primeiro estado no final de seis vibrações relativas ao ponto $B$, ou as quatro vibrações relativas ao ponto $C$ ou as duas vibrações relativas ao ponto $D$. Mas segundo a maneira que os srs. d'Alembert e Euler consideram a coisa, toda esta mistura de vibrações seria apenas duas vibrações simples. Nesse exemplo não acontecerá que os três pontos $A, B$ e $C$ se reúnam ao ponto fixo $D$. Mas se se supõe as três quantidades arbitrárias $\sqrt{a}, \sqrt{b}$ e $\sqrt{c}$, estar em razão de 3,5 , e 15 , os tempos de vibração estarão na mesma razão, e os pontos $A, B$ e $C$ se encontrarão reunidos ao ponto $D$ no final de cada semi-vibração do ponto $C$; deste modo as excursões serão perfeitamente semelhantes e iguais de cada lado do ponto fixo $D$ : mas, na minha opinião, o ponto $A$ deve [ser considerado] fazer cinco semi-vibrações antes que isso aconteça, ao passo que no outro sentido ele deve fazer uma única semi-vibração. Para ver a necessidade de considerar esses movimentos recíprocos em conformidade com a nossa teoria, há que se supor as distâncias arbitrárias iniciais $B C$, e $C D$, extremamente pequenas em relação à $A B$; haverá, portanto, somente as vibrações $A B$ que sejam sensíveis e o corpo $A$ fará manifestamente cinco semi-vibrações antes da junção perfeita dos pontos $A, B$ e $C$ ao ponto $D$. Mas, se se considerasse as distâncias $A B$, e $B C$, como extremamente pequenos com relação à $C D$, haverá somente as vibrações $C D$ que sejam sensíveis, e o corpo $A$ não poderia mais [ser considerado] ter feito uma única vibração. É necessário portanto evitar aqui todo equívoco nas palavras vibração e isocronismo. Se as quantidades $\sqrt{a}, \sqrt{b}$ e $\sqrt{c}$ são incomensuráveis, não poderá jamais acontecer que o sistema retome o seu estado inicial, nem que o corpo $A$ retornaria ao ponto de onde ele tinha partido, uma vez que os pontos $A, B$ e $C$ não terão jamais seus mais compridos alongamentos relativos em um mesmo instante: entretanto cada espécie de vibração relativa se fará separadamente segundo a lei dos pêndulos simples cicloidais. Essa é a fonte da razão que impede de entender a nova teoria dos srs. d'Alembert e Euler a todos os tipos de corda, qualquer lei de desigualdade que possa ter na sua espessura. 
XXII. Na aplicação dessas reflexões às cordas vibrantes será necessário supor $b=\frac{1}{4} c, a=\frac{1}{9} c$ etc. de sorte que os números de diferentes espécies de vibrações simples estejam em razão de números naturais 1,2 e 3 etc. começando por aquele do ponto $C$ relativo ao ponto fixo $D$, em seguida aquele do ponto $B$ relativo ao seu centro de força móvel $C$, e então aquele do ponto $A$ relativo ao centro de força móvel $B$.

XXIII. Suponhamos agora a distância inicial $A B=\alpha, B C=\beta, C D=\gamma$ etc. e que ao final de um tempo dado $t$ a distância $A B$ seja tornada $x$, a distância $B C=y$, a distância $C D=z$ etc.; seja também a velocidade relativa entre os pontos $A$ e $B=u^{\prime}$, aquela entre os pontos $B$ e $C=u^{\prime \prime}$, aquela entre os pontos $C$ e $D=u^{\prime \prime \prime}$ etc, ter-se-á

$$
\begin{aligned}
& d u^{\prime}=\frac{x}{a} d t \text { e } u^{\prime}=\sqrt{\frac{\alpha \alpha-x x}{a}} \\
& d u^{\prime \prime}=\frac{y}{b} d t \text { e } u^{\prime \prime}=\sqrt{\frac{\beta \beta-y y}{b}} \\
& d u^{\prime \prime \prime}=\frac{z}{c} d t \text { e } u^{\prime \prime \prime}=\sqrt{\frac{\gamma \gamma-z z}{c}}
\end{aligned}
$$

Além dessas equações a geometria nos ensina ainda acerca de construções e equações entre o tempo $t$ e as distâncias $x, y, z$ etc. Pois, seja o seno total expresso pela unidade e que se denota por $A \cdot \cos F$ um arco de círculo, cujo raio é um, e cujo cosseno é uma certa quantidade $F$, poder-se-á exprimir o tempo $t$ por cada uma das equações seguintes

$$
\begin{aligned}
& t=\sqrt{a} \times A \cdot \cos \frac{x}{\alpha} \\
& t=\sqrt{b} \times A \cdot \cos \frac{y}{\beta} \\
& t=\sqrt{c} \times A \cdot \cos \frac{z}{\gamma}
\end{aligned}
$$

Por meio destas equações poder-se-á determinar cada uma das distâncias $x, y, z$ etc pelo mesmo tempo $t$ comum convertendo os sinais; dessa forma ter-se-á

$$
\begin{aligned}
& x=\alpha \cos A \cdot \frac{t}{\sqrt{a}} \\
& y=\beta \cos A \cdot \frac{t}{\sqrt{b}} \\
& z=\gamma \cos A \cdot \frac{t}{\sqrt{c}}
\end{aligned}
$$

Nessas equações é necessário entender por $\cos A \frac{t}{\sqrt{a}}$ o cosseno de um arco de um círculo, que é igual a $\frac{t}{\sqrt{a}}$ e cujo raio é igual à unidade, e assim os outros. Além disso, as 
quantidades $\sqrt{a}, \sqrt{b}, \sqrt{c}$, etc. marcam os tempos de um corpo animado pelo peso natural empregado para cair livremente de alturas $\frac{1}{2} a, \frac{1}{2} b, \frac{1}{2} c$, etc.

Nós determinamos, portanto, cada uma das quantidades $x, y, z$ e etc. pelo mesmo tempo $t$, e por consequência a distância absoluta do corpo ao último ponto fixo, cuja distância é $x+y+z+e t c$. Nós determinamos semelhantemente para cada instante as velocidades relativas $u^{\prime}, u^{\prime \prime}, u^{\prime \prime \prime}$, etc e a velocidade absoluta do ponto $A$ expressa por $u^{\prime}+u^{\prime \prime}+u^{\prime \prime \prime}+$ etc . assim como os elementos de todas essas velocidades.

XXIV. Poder-se-á, no lugar dos tempos expressos por $\sqrt{a}, \sqrt{b}, \sqrt{c}$, etc. considerar os tempos que respondem a uma vibração simples relativa do ponto $A$ com relação ao ponto $B$, e depois do ponto $B$ com relação ao ponto $C$, em seguida do ponto $C$ em relação ao ponto $D$. Se nós designamos, portanto, esses tempos por $\theta^{\prime}, \theta^{\prime \prime} \mathrm{e}$ $\theta^{\prime \prime \prime}$ e que $\pi$ seja a semicircunferência de um círculo que possui por raio a unidade, será necessário supor nas equações do artigo precedente $\sqrt{a}=\frac{\theta^{\prime}}{\pi}, \sqrt{b}=\frac{\theta^{\prime \prime}}{\pi}, \quad \sqrt{c}=\frac{\theta^{\prime \prime \prime}}{\pi}$, substituições após as quais todas as quantidades tornam-se manifestamente homogêneas.

Seja, por exemplo, $\theta^{\prime}=1^{\prime \prime}, \theta^{\prime \prime}=2^{\prime \prime}, \theta^{\prime \prime \prime}=3^{\prime \prime}$, e seja questão de determinar todas as circunstâncias do movimento fazendo $t=$ um quarto de segundo; eu digo que se terá $x=\alpha \cos A \frac{\pi t}{\theta^{\prime}}=\alpha \cos A \frac{1}{4} \pi=\alpha \sqrt{\frac{1}{2}} ; y=\beta \cos A \frac{\pi t}{\theta^{\prime \prime}}=\beta \cos A \frac{1}{8} \pi=\beta \cos 22^{\circ} 30^{\prime}$; e $z=\gamma \cos A \frac{\pi}{12}=\gamma \cos 15^{\circ}$; portanto $x+y+z=0,70710 \alpha+0,92387 \beta+0,96592 \gamma$, quantidade [esta] que marca a distância do corpo $A$ ao ponto $D$ após um quarto de segundo de tempo. Ter-se-á, em seguida, $u^{\prime}=\sqrt{\frac{\alpha \alpha-x x}{a}}=\frac{\alpha}{\sqrt{a}} \operatorname{sen} A \frac{1}{4} \pi$; $u^{\prime \prime}=\sqrt{\frac{\beta \beta-y y}{b}}=\frac{\beta}{\sqrt{b}} \operatorname{sen} A \frac{1}{8} \pi=\frac{\beta}{2 \sqrt{a}} \operatorname{sen} A \frac{1}{8} \pi, \quad$ e $u^{\prime \prime \prime}=\frac{\gamma}{3 \sqrt{a}} \operatorname{sen} A \frac{1}{2} \pi . \quad$ Disso resulta para nosso exemplo a velocidade absoluta $u^{\prime}+u^{\prime \prime}+u^{\prime \prime \prime}=\frac{6 \alpha \operatorname{sen} 45^{\circ}+4 \beta \operatorname{sen} 22^{\circ} 30^{\prime}+2 \gamma \operatorname{sen} 15^{\circ}}{6 \gamma a}$.

XXV. Mas, sem nos determos a este tipo de determinações, que não têm muita relação com aquilo que eu propus como principal, examinamos sobretudo sobre quais circunstâncias a velocidade absoluta $u^{\prime}+u^{\prime \prime}+u^{\prime \prime \prime}$ pode tornar-se 0 , nos restringindo às três espécies de vibrações simples juntamente misturadas. Será necessário para este efeito, em virtude do §. 23, fazer $\sqrt{\frac{\alpha \alpha-x x}{a}}+\sqrt{\frac{\beta \beta-y y}{b}}+\sqrt{\frac{\gamma \gamma-z z}{c}}=0$, substituímos para $x$, $y$,e $z$ seus valores em $t, \quad$ e nós teremos $\frac{\alpha}{\sqrt{a}} \operatorname{sen} A \frac{t}{\sqrt{a}}+\frac{\beta}{\sqrt{b}} \operatorname{sen} A \frac{t}{\sqrt{b}}+\frac{\gamma}{\sqrt{c}} \operatorname{sen} A \frac{t}{\sqrt{c}}=0$. Eu me proponho a determinar o valor de $t$, que satisfaça a essa equação em algum exemplo particular. Nós suporemos, portanto, $\theta^{\prime \prime \prime}$ ou $\pi \sqrt{c}=2 \theta^{\prime \prime}$ ou $2 \pi \sqrt{b}=3 \theta^{\prime}$ ou $3 \pi \sqrt{a}$, quer dizer nós suporemos, que, enquanto 
o ponto $C$ faz uma vibração para o ponto fixo $D$, o ponto $B$ faça duas para o ponto $C$, e o ponto $A$ faça três para o ponto $B$, suposição que demanda $\sqrt{c}=2 \sqrt{b}=3 \sqrt{a}$. A nossa expressão acima dará

$$
3 \alpha \operatorname{sen} A \frac{3 t}{\sqrt{c}}+2 \beta \operatorname{sen} A \frac{2 t}{\sqrt{c}}+\gamma \operatorname{sen} A \frac{t}{\sqrt{c}}=0 .
$$

Seja agora $A \frac{t}{\sqrt{c}}=q$, e ter-se-á $\operatorname{sen} A \frac{2 t}{\sqrt{c}}$, quer dizer o seno do arco duplo $2 q \sqrt{(1-q q)}$ e $\operatorname{sen} A \frac{3 t}{\sqrt{c}}=3 q-4 q^{3}$, e a expressão de cima nos fornece

$$
3 \alpha\left(3 q-4 q^{3}\right)+2 \beta \times 2 q \sqrt{(1-q q)}+\gamma q=0
$$

ou

$$
9 \alpha-12 \alpha q q+4 \beta \sqrt{(1-q q)}+\gamma=0
$$

Pela primeira destas equações já se vê que se pode fazer $q=\operatorname{sen} A \frac{t}{\sqrt{c}}=0$, o que fornece $z=\gamma \cos A \frac{t}{\sqrt{c}}= \pm \gamma ; y=\beta \cos A \frac{t}{\sqrt{b}}=\beta \cos A \frac{2 t}{\sqrt{c}}=\beta$, e $x= \pm \alpha$, e por consequência $x+y+z= \pm \alpha+\beta \pm \gamma$. Este é o caso que se oferece; mas existem ainda outros indicados para a segunda equação, que podem ser bem reais. Para encurtar o cálculo, nós nos limitaremos aos casos que fazem $9 \alpha+\gamma=4 \beta$; e nesses casos encontram-se $\operatorname{sen} A \frac{t}{\sqrt{c}}$ ou

$$
q= \pm \frac{\sqrt{(6 \alpha \beta-\beta \beta)}}{3 \alpha}
$$

e em seguida o $\cos A \frac{t}{\sqrt{c}}$ ou

$$
\sqrt{(1-q q)}= \pm \frac{\sqrt{(9 \alpha \alpha-6 \alpha \beta+\beta \beta)}}{3 \alpha}= \pm\left(1-\frac{\beta}{3 \alpha}\right) .
$$

Daí, obtém-se $z= \pm \gamma\left(1-\frac{\beta}{3 \alpha}\right)$,

este valor de $z$ já marca a posição do ponto $C$; encontrar-se-á em seguida o valor de $y$, e enfim aquele de $x$, e por aí ter-se-á determinado as posições dos três pontos $C, B$ e $A$. Vê-se, portanto, que a nossa questão é muitas vezes a mais suscetível de soluções bem reais, apesar de que elas não o sejam sempre: pois no caso presente é necessário que a quantidade $\frac{\beta}{3 \alpha}$ seja sempre um número positivo, e que $\beta$ seja menor que $6 \alpha$.

Desçamos aqui a um exemplo bem particular: suponhamos $\beta=4 \alpha$ e por consequência $\gamma=7 \alpha$. Nesse caso é necessário tomar $\sqrt{(1-q q)}$ negativa, e fazer $\sqrt{(1-q q)}=\frac{-1}{3}$, o que dá o arco $\frac{t}{\sqrt{c}}$ de $109^{d}, 28^{\prime}$, cujo cosseno é $-\frac{1}{3}$, o arco $\frac{2 t}{\sqrt{c}}$ de $218^{d}, 56^{\prime}$, cujo cosseno 
é $-0,77787$; e o arco $\frac{3 t}{\sqrt{c}}$ de $328^{d}, 24^{\prime}$, cujo cosseno é 0,83172 . Estes valores dão $z=\frac{-1}{3} \gamma=\frac{-7}{3} \alpha ; \quad y=-0,77787 \beta=-3,11148 \alpha ; \quad$ e $x=0,85172 \alpha, \quad$ e $\quad$ por consequência $x+y+z=-4,59309 \alpha$. Assim, a distância inicial do corpo $A$ desde o ponto $D$ sendo expressa por 12, existirá ainda duas posições do corpo $A$, nas quais a velocidade absoluta seja nula, e as distâncias ao ponto $D$ serão, portanto, $-4 \mathrm{e}$ $-4,59309$.

XXVI. Aqui está agora a natureza do movimento absoluto do corpo $A$ com relação ao ponto fixo $D$; a figura 8 marca os pontos principais com a justa proporção de distâncias. Eu digo, portanto, que o corpo partindo do ponto $A$ será primeiramente acelerado e em seguida retardado, após o que ele será parado por um momento no ponto $B$; ele retornará de lá até o ponto $C$, onde ele repousará ainda um instante; do ponto $C$ ele retornará em direção ao ponto $B$ parando lá de novo durante um instante, e do ponto $B$ enfim ele retornará ao ponto $A$, do qual ele tinha partido, onde sua velocidade será ainda nula, e portanto tudo será reduzido ao seu primeiro estado. Além disso, o tempo empregado para fazer a primeira vibração $A B$ é expresso por $109^{\circ}, 28^{\prime}$, o tempo para fazer a segunda pequena vibração $B C$ pelo arco $70^{\circ}, 32^{\prime}$, esse segundo tempo será ainda empregado para fazer a terceira vibração $C B$, e o primeiro tempo para fazer a quarta vibração $B A$. Se se expressa, portanto, todo o tempo, que o corpo emprega antes que se recoloque em seu primeiro estado por 21600 , a primeira vibração empregará 6568 da mesma maneira que a quarta, ao passo que cada uma das duas médias empregará 4232 . Como nesse exemplo a segunda e terceira excursão são bem pequenas, o movimento do corpo parecerá como quase em repouso durante quase a metade do tempo inteiro. Mas terse-ia podido escolher outros exemplos onde as excursões parciais seriam tornadas como iguais e como isócronas, e portanto é uma questão, se o corpo deve ser suposto ter feito quatro vibrações, ou duas; no sentido dos srs. d'Alembert e Euler, seria necessário sempre dizer, que ele só teria feito duas, embora se pôde fazê-lo cem idas e voltas todas quase iguais, e quase isócronas, e cujos movimentos far-se-iam quase inteiramente segundo as leis dos movimentos isócronos simples e ordinários.

XXVII. Isso que eu acabei de dizer acerca do corpo $A$, ocorre perfeitamente a cada ponto nas novas vibrações de cordas: eu me contentaria, para fazer ver essa conformidade inteira, em explicar a mistura dessas duas primeiras espécies de vibrações mais simples, expressas pelas duas primeiras figuras. Eu representei essa mistura pela sexta figura, a qual eu dei a construção no §. 8. Seja nessa curva absoluta ApaqB a maior ordenada relativa $p m=\rho$, a maior ordenada $a r$ da curva ideal $A m a n B=\sigma$; e seja ainda o comprimento da corda inteira $A B=a$; que se tome uma abscissa determinada $A b=x$ com sua ordenada $b d$; a partir disso ter-se-á em virtude da teoria do sr. Taylor $c d=\rho \operatorname{sen} \frac{2 \pi x}{a}$ e $b c=\sigma \operatorname{sen} \frac{\pi x}{a}, \quad$ e por consequência a ordenada $b d=\rho \operatorname{sen} \frac{2 \pi x}{a}+\sigma \operatorname{sen} \frac{\pi x}{a}$. Seja $b d=y, \quad$ ter-se-á, $\quad$ supondo $d x$ constante, 
$d d y=\left(\frac{4 \pi \pi}{a a} \rho \operatorname{sen} \frac{2 \pi x}{a}+\frac{\pi \pi}{a a} \sigma \operatorname{sen} \frac{\pi x}{a}\right) d x^{2} ;$ no entanto, nomeando $P$ o peso que tensiona a corda, e $p$ o peso da corda tensionada e uniforme, de fato, a força aceleradora absoluta do ponto $d$ é expressa por $\frac{P}{p} \cdot \frac{a d d y}{d x^{2}}$; essa força aceleradora será então aqui $\frac{P}{p} a\left(\frac{4 \pi \pi}{a a} \rho \operatorname{sen} \frac{2 \pi x}{a}+\frac{\pi \pi}{a a} \sigma \operatorname{sen} \frac{\pi x}{a}\right)$ ou $\frac{4 P}{p} \cdot \frac{\pi \pi \rho}{a} \operatorname{sen} \frac{2 \pi x}{a}+\frac{P}{p} \cdot \frac{\pi \pi \sigma}{a} \operatorname{sen} \frac{\pi x}{a}$, que faz a força aceleradora do ponto $d=\frac{4 P}{p} \bullet \frac{\pi \pi}{a} \bullet c d+\frac{P}{p} \bullet \frac{\pi \pi}{a} \bullet b c$. Nós vemos então, que a força aceleradora inteira é composta de duas partes, em que a primeira é proporcional à distância $d c$, e que faz fazer ao ponto $d$ vibrações isócronas relativas ao ponto $c$, que faz aqui um centro de força móvel: a segunda parte da força aceleradora do ponto $d$ é $\frac{P}{p} \bullet \frac{\pi \pi}{a} \bullet b c$ : no entanto, pela natureza da curva $A \operatorname{can} B$, o ponto $c$ é animado em direção ao ponto imóvel $b$ com a mesma força aceleradora $\frac{P}{p} \cdot \frac{\pi \pi}{a} \bullet b c$; assim a segunda força produz constantemente a mesma aceleração aos pontos $d$ e $c$, e só fará transportar a pequena distância $d c$ sem mudá-la de jeito nenhum. Nós estamos, portanto, inteiramente no mesmo caso que aqueles dos $\S \S .19$ e 20. Para obter uma identidade inteira será necessário supor na sétima figura $C D=o, A B=d c$ e $B C=c b$, ou $\alpha=\rho \operatorname{sen} \frac{2 \pi x}{a}$, $\beta=\sigma \operatorname{sen} \frac{\pi x}{a}$ e $\gamma=0$, e nessas expressões a quantidade $x$ é constante com relação ao movimento do mesmo ponto $d$. Assim o movimento de cada ponto na curva $A d a q B$ será com relação à curva ideal $A \operatorname{can} B$, o mesmo que o movimento absoluto da segunda figura, e o movimento absoluto da curva ideal $A$ can $B$ será o mesmo que aquele da primeira figura; assim a vibração segundo a figura a $\operatorname{AdaqB}$ não é absolutamente senão uma mistura de vibrações segundo a primeira e a segunda figura combinadas conjuntamente, em que cada espécie se faz independentemente uma da outra. Esse movimento absoluto do ponto $d$ abarca realmente dois movimentos periódicos, um com relação ao ponto $c$, e outro com relação ao ponto $b$; o número dos primeiros retornos periódicos será sempre o dobro daqueles dos segundos. O espírito percebe uma e outra espécie desses retornos periódicos e percebe com isso um som duplo, em que um é a oitava do outro. Como as pequenas quantidades $p m$ e $a r$ designadas por $\rho$ e $\sigma$ podem ter alguma relação, só observar-se-á a primeira espécie de movimento periódico fazendo $p m$ muito maior que $a r$, enquanto que só sentir-se-á a segunda espécie fazendo ar muito maior que $p m$; no primeiro caso, o tempo de uma vibração é manifestamente a metade menor que o segundo, enquanto que segundo a maneira de considerar as vibrações dos srs. d'Alembert e Euler, o tempo de cada período é sempre o mesmo, qualquer razão que haja entre $\rho$ e $\sigma$, isso que não me parecia conforme o princípio de continuidade. Pois, se o tempo de uma vibração é segundo eles sempre $t$, qualquer razão que houvesse entre $\rho$ e $\sigma$, porque ele torna manifestamente $\frac{1}{2} t$ fazendo $\rho=0$ ? Para responder a esta dificuldade, me parece que é preciso necessariamente dizer, que ele faz ao mesmo tempo uma dupla espécie de vibração 
na corda, e que a mistura dessas duas espécies forma aquilo que esses geômetras chamam vibrações simples; que a segunda espécie diminui à medida que se diminui a quantidade $\sigma$ , e que ela se torna nula fazendo $\sigma=0$; e, portanto, não haverá qualquer descontinuidade.

XXVIII. Eu espero que aquilo que eu acabei de dizer nesse Mémoire poderá servir para propagar luz sobre a natureza das novas vibrações das cordas, encontradas com tanta sagacidade pelos srs. d'Alembert e Euler; e isso era todo meu objetivo. Se o método em que eles se fizeram servir para resolver seus problemas é muito mais difícil que o meu, eu não me admiro com a vantagem da superioridade do gênio deles. Quanto à questão se as novas vibrações são realmente vibrações simples e síncronas para todos os pontos, onde elas não são sobretudo uma mistura de várias diferentes vibrações coexistentes em uma mesma corda e todas de diferentes durações, eu só falei para melhor explicar a natureza dessas vibrações, estando bem distante de fazer uma querela com estes também grandes homens sobre o significado de certos termos.

Eu tratarei em um segundo Mémoire de alguns problemas novos, em que a solução resulta bem facilmente de nossos princípios e que ao mesmo tempo coloca esta matéria em uma nova luz. 


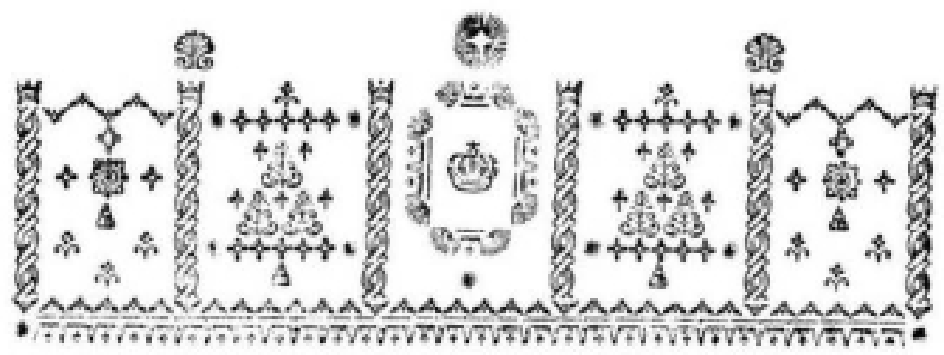

\section{R E F L E X I O N S}

E T

\section{E C L A I R C I S S E M E N S}

SUR LES NOUVELLES VIBRATIONS DES COR.

DES EXPOSEES DANS LZS MEMOIRES

DE L'ACADÉMIE

de 1747 . \& 1748 .

PAR M. DANIEL BERNOULLI.

$0+\int^{-1}$

Mr.

I.

M. Taylor eft parvenu le premier à connoitre le nombre des vibrations, que fait dans un tems donné une corde uni-

A formement epaifte d'une longueur donnée, d'un poids donné, \& tenduẽ par une force donnée. Il n'étoit pas poifible de déterminer ce nombre fans connoitre préalablement les courbures, que prendroient les cordes pendant tout le tems que dureroient leurs vibrations; il a done démontré, que cette courbure étoit 


\section{数 $\quad 4_{4} 8$ 象}

conftamment la compagne d'une cycloïde extremement allongée, dans laquelle les appliquées repréfentent les finus des arcs reprélentés par les abfciffes: aufli n'elt-ce à mon avis que fous cette forme que les vibrations petivent devenir régulieres, fimples, \& ifochrones, malgré l'inégaiité des excurfiuns. Avec cetre idśe, que $j$ ai toujours euẻ, je ne pouvois qu’ètre furpris de voir dans lss Mémoircs des années 1747 . \& 1748. une infinité d'autres courbures comme douécs de la même propriétí; il ne me falloit pas moirs que les grands noms de Mrs. d' $A$. lembert \& Euler; que je ne pouvois foupçonner d'aucune inattention, pour examiner s'il n'y auroir pas quelque équivo fue dans l'aggréga. tion de toutes ces courbes avec ceile de M. Taylor, \& dans quel fens on pourroit les admertre. J'ai vủ aufli- tôt, qu'on ne pouvoit åłmettre cetre mulritude de courbes que dans un fens tout - à- fait inpropre; je n'en eftime pes moins les calculs de Mrs. d'Alembert \& Eaír, qui renferment certainement tout ce que l'Analyfe peut avoir de plus profond \& de plus fublime; mais qui montrent en même tems, qu'une ana. Jyfe abftraite, qu'on écoure fans aucun examen fyntherique de la queftion propolée, eft fujetre à nous furprendre plutôt qu'ù nous éclairer. Il me femble à moi, qu'il n'y avoit qu'à faire artention à la narure des vibrations fimples des cordes, pour prévoir fans aucun calcul tour ce que ces deux grands Géometres ont trouvé par les calcu's les plus épineux \& les plus abftraits, dont l'efprit analytique fe foit encore avifé

II. Remarquons d'abord que, fuivant la théorie de M. Taylor, une corde tenduë peut former fes vibrations uniformes d'une infinité de manicres, qui different entre elles pour le phyfique, mais qui reviennent au même pour le géometrique, parce que dans chacune de ces manieres on ne fait que changer l'unité qui fert de mefure. Ces ma. nieres differentes confiftent dans le nombre des ventres, que la corde peut former durant fes vibrations. Quand il n'y a qu'un feul ventre, comme dans la premiere Figure, les vibrations font les plus tardives, elles formen le fon fondamental; quand il y a deux venures, \& un

noeud 


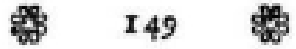

noeud au milieu de Paxe, les vibrations fe doublent, \& elles forment l'octave du fon fondamental; lorsque la corde forme trois, quatre, ou cing ventres, avec deux, trois, ou quatre nocuds, par des diftances muruelles égales, comme dans les figures 3. $4 . \&$ s. les vibrations fe triplent, quadruplent, ou quintuplent, \& forment la douzième, la double octave, ou la rierce majeure de la double octave, rélativement au fon fondamental. Cette multiplicité va à l'infini. Dans chaque cfpece de ces vibrations les excurfions totales peuvent être grandes ou petites à difcrétion, pourvû que les plus grandes puiffent ètre cenfées extrèmement petites. La nature de ces vibrations eft relle que, non feulement chaque point commence \& finit chaque vibration fimple an même inftant, mais encore que tous les points fe mettent après chaque demi-vibration fimple dans la polition de l'axe $\mathrm{AB}$. It faut confidérer toutes ces conditions comme effentielles, \& tout aufli-tôt il n'y a que les courbes données par M. Taylor, qui farisfaffent au problème. Mais en féparant ces conditions on peut former une infinité de courbes, qui fatisfafent à quelque condition féparément; mais je ferai voir combien peu on feroit fondé en ces cas à appeller les vibrations ifochrones pour chaque point. Il en eft de ces vibrations, com. me des mouvemens réciproques des corps, qui defcendent \& montent alternativement fur une courbe ; certe courbe ne peut ĉrre que la cycloïde, fillon demande que toutes les defcentes, grandes ou petites, foyent ifochrones entre elles de méme que les montées; mais fi l'on veut fimplement que les vihrations entieres foyent ifochrones entre elles, on peut donner autant de courbes qu'on demande, qui fatisfaffent à ce problème; puisque j’ai demontré dans les Mémoires de Petersbourg, que quelle courbe de defcente on donne, on peut toujours déterminer la courbe de montée, telle que les deux rems cmployés à la defcente $\&$ ì la montée pris enfemble faffent une mểme fomme, quelque inégalité qu'il y air entre les amplitudes des excurfions.

III. Si j'ai dit que les cordes peuvent faire leurs vibrations fimples d'une infunité de manieres, dont les cing premieres figures fervent 


\begin{abstract}
Iso 䢿
d'exemples, ce n'eft pas là une vérité abftraite feulement; on peut la rendre réelle, comme lexemple des trompettes marines le montre affez par les fons qu'on en tire : les Expériences qu'on fait en plaçant un chevalet dans quelqu'un des points $a$, \& en pinçant le bout $A a$, confirment la même chofe ; puisqu'elles nous aprennent, qu il fe forme aux points $b, c, d, \& c$. des noeuds à diftances égales, qqui demeurent comme en repos, pendant que tous les autres points font agités. Cette multiplicité infinie de vibrations fe manifefte dans tous les corps fonores, de quelque nature qu'ils puiffent êrre; c'eft par là qu'on peut tirer des cors de chaffe, des trompettes, \&c. rous les fons qui vont en progreffion des nombres naturels; c'eft à dire dans la même pro. greffion que les fons, qu'on peut tirer d'une feule \& même corde par fes differentes efpeces de vibrations. En fermant tous les trous d'unc flutc traverfiere, on peut par la fimple variarion de l'embouchure obtenir d'abord le fon le plus bas, ou fondamental, \& puis fucceffivement fon oftave, fa douzième, fa double octave, fa dix-feptième majeure, qui font pareillement comme $t, 2,3,4$ \& 5 . mais il ne faut pas croire pour cela, que cetre progreffion foit généralc. Aprés m'èrre formé ane bonne théorie fur les vibrations de l'air dans les inftrumens à vent, $\mathrm{j}$ 'en ai conclu, qu'on ne pourroit tirer des tuyaux bouchés que les fons, qui vont en progreffion des nombres impairs, favoir $\mathrm{r}, 3,5$, 7 , \&c. \& ma conclufion a été confirmée parl'Expérience : car ayant ôté la piece d'en - haut d'une flùte traverfiere, \& la bouchant avec la main, j'en ai tiré d'abord le fon le plus bas, \& puis renforçant le foufle fa douzième fans paffer par l'octave, enfuite fa dix-feptième majeure, \& enfin un ton qui n'eft pes reçu dans la Mufique, \& qui approchoit de la vingt - unième du fon fondamental. Je ne fai fi cette propriété a déja été remarquée par d'autres ; mais elle me paroit d'autant plus remarquable, qu'elle eft peut - être propre à expliquer en quoi confiftent ces accès de facile réfléxion \& de facile transmiffion des rayons, obferves avec tant de fagaciré par le grand Newton. C'eft fur quoi je compte de donner un Mémoire, quand j'aurai expliqué \& réduit au oalenl les
\end{abstract}

vibra. 


\begin{abstract}
I-9. 8
vibrations de l'air formées dans des tuyaux ouverts \& bouchés, \& démontré l'analogie entre ces vibrations de l'air \& celles de l'ether, qui fait la lumiere. Je n'ai fait ici cetre remarque, que pour prouver, que les differens fons tirés da même corps fonore ne vont pas toujours dans la progrelfion des nombres naturels. Mais je dis plus : ces fons peuvent avoir telle proportion, qu'il n'y a aucune formule en quantités finies qui puiffe l'exprimer; comme on peut voir par les fons, que j'ai calculés autrefois, qui font ceux qu'on peut tirer d'une verge d'acier frappée par des petirs coups. C'étoit un problème nouveau, \& qui demandoit beaucoup de circonfpection; aprèsl’avoir réfolu, je l'ai pro. pofé à M. Euler, qui en a donné une folution parfaitement conforme à la mienne, quoique incomplete d'abord en ce qu'il avoit omis la moitié des fons poffibles; je l'en ai averti, \& il y a fuppléé dans les Actes de Leipfig.

IV. Ma conclufion eft, que tous les corps fonores renferment en puiffance une infinité de fons, \& une infinité de manieres correfpondantes de faire leurs vibrarions régulieres; enfin, que dans chaque differente efpece de vibrarions les infléxions des parties du corps fonore fe font d'une maniere differente. Mais ce n'eft pas de cette multitude de vibrations appliquée aux cordes tenduës, que $\mathrm{Mr}$. d'Alembert \& Euler prérendent parler; elle n'étoit pas inconnuẻ à M. Taylor : ils multiplient à l'infini chaque efpece, en accordant à chaque intervalle entre deux noeuds voifins une infinité de courbures relles que chaque point commence \& acheve aux mêmes inftans fes vibrations, pendant que, fuivant la théorie de $\mathrm{M}$. Taylor, chaque dit intervalle doit néceffairement prendre la feule courbure de la compagne de la cycloìde extrémement allongée. Cette contradiction apparente entre d'aufi grands Géometres me paroir demander quelque éclairciffement.

V. Remarquons done, que la corde $\mathrm{AB}$ peut non feulement haire fes vibrations fuivant la figure premiere, ou feconde, ou troifième, \& ainfi à l'infini, mais qu'il peut fe faire encore un mélange de toutes ees vibrations avec toutes les combinaifons poffibles; \& cependant

toutes
\end{abstract}




\section{数 152 क्षै}

toutes les nouvelles courbes \& nouvelles efpeces de vibrations données par Mrs. d'Alembirt \& Exler ne font abfolument qu'un mélange de plufieurs efpeces de vibrations Tayloriennes. Si cela eft vrai, je ne faurois approuver l'aggrégation de toutes ces nouvelles courbes; puisque la corde en s'y conformant ne donne pas un feul \& metme ton, mais plufieurs tons à la fois. Pour fentir l'incongruilté de cette nouvelle aggrégration, fuppofons, au lieu d'une feule corde, cinq cordes par exem. ple, entierement égales en tout fens; que la premiere fafte fes vibrations fuivant la loi de la premiere figure; la feconde corde fuivant la loi de la feconde figure, \& ainfi des autres. Suppofons que toutes ces cordes commencent leurs vibrations dans un même inftant; voudroiton appeller ces cinq efpeces de vibrations ifochrones entre clles, fim. plement parce qu'elles finiffent une de leurs vibrations au même instant que la premicre corde finit chacune des fiennes? Non fans doute, parce que la feconde corde fait deux vibrations, la troifième trois, \& ainfi des autres, pendant que la premiere corde n'en fait qu'une; \& que chaque corde donne un ton different à proportion. Cependant tout ce que je viens de dire par rapport à plufieurs cordes entierement égales, peut \& doit être appliqué à une feule \& même corde.

VI. Effectivement tous les Muficiens conviennent, qu'une longue corde pincée donne en même tems, outre fon ton fondamental, d'autres tons beaucoup plus aigus ; ils remarquent fur tout le mêlange de la douzième \& de la dix-feptième majeure : s'ils ne remarquent pas auffi diftinctement l'octave \& la double octave, ce n'eft qu'à caufe de la trop grande reffembiance de ces deux tons avec le ton fondamental. Voilà une preuve évidente, qu'il peut fe faire dans une feule \& même corde un mêlange de plufieurs fortes de vibrations Tayloriennes à la fois. On entend pareillement dans le fon des groffes cloches un mèlange de tons differens. Si l'on tient par le milieu une verge d'acier, \& qu'on la frappe, on entend à la fois un mêlange confus de plufieurs tons, lesquels étant appréciés par un habileMuficien fe trouvent extré-

mement 


\section{承 $153 \quad$ 急}

mement desharmonieux, de forte qu'il fe forme ici un concours de vi. brations, qui ne commencent \& ne finiftent jamais dans un même instant, finon par un grand hazard : d'où l'on voit que l'harmonie des fons, qu'on entend dans une même corps fonore à la fois, n'eft pas effentielle à cette matiere, \& ne doit pas fervir de principe pour les fyftèmes de Mufique. L'air n'eft pas exemt de cetre multiplicité de fons coẽxiftans: ilarrive fouvent qu'on tire deux fons differens à la fois d'un tuyau; mais, ce qui prouve le mieux, combien peu les differentes ondulations de l'air s'entre - empêchent, eft qqu'on entend distinctement toutes les parties d'un concert, \& que toutes les ondulations caufées par ces differentes parties fe forment dans la même maffę d'air fans fe troubler mutuellement, tout comme les rayons de la lumiere, qui entrent dans une chambre obfeure à travers une petite ouverture, ne fe troublent point.

VII. Après ces remarques il fera facile de conftruire une infinité de courbes initiales à la corde $\Lambda \mathrm{B}$ avec cette condition, que chaque point de la corde arrive quelquefois en même tems à un point de repos inftantané, \& de donner la loi générale pour toutes ces courbes fans aucun calcul prćalable. Commençons par la combinaifon des deux premieres figures. Suppofons que la corde faffe fes vibrations pour former le fon fondamental conformément à la premiere figure; cette courbure étant cenfée infiniment petite, la corde pourra encore être confidérée comme une ligne droite, \& $\mathrm{fa}$ courbe pourn fervir d'axe mobile à la courbe de la feconde figure, à deux branches; \& de là il réfulte une nouvelle courbe, qui remplira la condition préfcrite. Voici done la conftruction de cette nouvelle courbe.

VIll. Soir (Fig. 6.) A m an B la courbure de la premiere figure: qu'on confidére cette courbe comme un axe droit, fur lequel on conftruife $\mathrm{A} p a q \mathrm{~B}$, entiérement la même par rapport à fon axe courbé que celle de la figure feconde, par rapport à l'axe parfaitement droit A B ; \& cette courbe A p aq B fera telle qu'on defiroit, \& la plus fimple des courbes données par Mir. d'Alembert \& Ealcr.
Nive. di l'Ais, Tum. IX,
$\mathrm{V}$
Comme 


\section{垂 154}

Comme la plus grande appliquée $p m$ peut avoir un rapport quel. conque à la plus grande appliquée $a r$, il cft clair, que cette courbe Apaq B contient déjà une infinité d'efpeces. Voici i préfent les propriétés de cette courbe A $a q \mathrm{~B}$.

(a) Je dis que la courbe idéale $\mathrm{A} m a n \mathrm{~B}$ fera fes vibrations par rapport à l'axe droit $A r b$, entierement fuivant la loi des vibrations fimples de la premiere figure.

(b) Enfuite, que chaque point de la courbe A paq B aura fon mouvement rélatif par rapport à chaque point correfpondant de la courbe $\mathrm{A} m a n \mathrm{~B}$, le même que le mouvement abfolu repréfenté par la feconde figure.

(c) Or chaque point de la feconde figure fair précifément deux vibrations, pendant que le même point en fait une dans les vibrations de la premiere figure. Il faut donc que tous les points de la courbe A $a_{q}$ B finiffent leurs vibrationsalternes au mème inftant, que ceux de la figure $\mathrm{A}$ ma $n \mathrm{~B}$ finiffent chacune des leurs.

(d) Lorsque, au commencement, un point de la courbe $\mathrm{A}$ paq B eft en dehors de la courbe $\mathrm{A}$ man $\mathrm{B}$, ce même point au bout de fes deux vibrations fera en dedans de certe derniere courbe, qui n'aura fait qu'une feule vibration; \& ainfi la courbe $A$ m $a n \mathrm{~B}$ ayant pris la fituation $\mathrm{A} m^{\prime} a^{\prime} n^{\prime} \mathrm{B}$, il arrivera que la courbe A $a q \mathrm{~B}$ prenne la fituation $A p^{\prime} a^{\prime} q^{\prime} \mathrm{B}$, \& que la courbe $\mathrm{A} p^{\prime} a^{\prime} q^{\prime} \mathrm{B}$ foit tout à fait la même qu'elle avoit été, en changeant fimplement l'ordre des côtés, c'eft à dire, la même que $\mathrm{B}_{\mathcal{Z} a p} \mathrm{~A}$, ce qui fait un cas du beau théoreme de M. Euler.

IX. De la même façon que nous avons combiné les vibrations de la premiere, \& de la feconde figure, on pourra combiner les vibrations de la premiere figure, avec celles de toute autre figure fans exception 


\section{t 155}

exception à l'infini; toures ces combinaifons peuvent metme fubfifter la fois ; ainfi; par exemple, la courbe abfoluè $\mathrm{A} p a q \mathrm{~B}$ de la fixième figure, pourra encore être confidérée comme une courbe translatrice, on pourra la partager en trois parties égales, \& y appliquer la même courbe qui, dans la troifième figure, eft appliquée à l'axe droit AB. Quelle que foit la courbe abfoluẽ réfultante de toutes ces combinaifons faites à difcrétion, il arrivera toujours que tous les points de la courbe arrivent dans un même inftant au point de repos inftantané du côté oppofé de l'axe, \& la courbe dans cet érąr fera toujours la même, en fituation renverfée, qu'elle avoit été au commencement, comme le dit M. Euler.

$X$. Si l'on ne combine enfemble que les figures, qui ont un nombre de ventres impair, ces courbes auront encore cette propriété, que chaque point de la courbe abfoluë réfultante paffe dans un même inftant par l'axe droit $A B$, ce qui peut par conféquent fe faire d'une infinité de façons; mais aufti-tôt qu'une feule courbe à ventres pairs s'y mêle, cela n'arrive plus. Voilà un expofé̉ phyfique des nouvelles vibrations des cordes données par Mrs. d'Alembert \& Evler; \& fi j’ai bien compris leurs énoncés, toures les nouvelles courbes qu'ils donnent, font comprifes dans notre conftruction, \& font un fimple melange de plufieurs efpeces de vibrations, dont chacune à part fe fait fuivant les loix décrites par M. Taylor. Mais il me femble que ce n'eft lì qu'une efpece de compofition de mouvement, qui ne peut donner aucune amplification à la théorie de $\mathrm{M}$. Taylor.

XI. Pour mieux fentir l'incongruïté d'une telle amplification, nous combinerons la courbe fondamentale de M. Taylor, qui eft repréfentée par la premiere figure, avec la figure anguiforme Taylorienne qui auroit 1001 ventres; il en réfultera une courbe du nombre des nouvelles courbes, qui aura cette propriété, que tous les points commençans au même inftant leur vibration, pafferont tous dans un même inftant par l'axe droit, \& arriveront dans un même inftant au

$$
\mathrm{V}_{2} \quad \text { point }
$$




\section{क 156 题}

point de repos, de l'autre côté de l'axe, pour recommencer un nouveau mouvement pareil au précedent. Mais qu'atrive- $t$ - il pendant ce mouvement? Je dis quil y aura dans la corde précifément 1000 points à de petites diftances égales, dont chacun fait une vibration, abfolument de la même maniere que fi les vibrations fe faifoient fimplement fuivant la loi de la premiere figure, pendant que tous les autres points feront $\mathrm{r} O 0 \mathrm{t}$ allées \& venuẻs, ou $\mathrm{t} 00 \mathrm{I}$ vibrarions, \& pourront même paffer soor fois l'axe droit. Ces roor vibrations feront enticres \& parfaires; dans chacune de ces vibrations chaque élément anra un moment de repos abfolu \& parfait, \& un moment auquel fa vitefle foit la plus grande. Si l'on vouloit confondre ces petires vibrations rapides avec la fimple vibration fondamentale, uniquement parce que la mille \& unième petite vibration finit au même inftant dans chaque point, que la vibration fondamentale finit, ce feroit appeller fynchrones deux pendules fimples, dont l'un auroit $I$ pied de longueur \& l'autre 1002001 pieds, parce que ces deux pendules, commençans leurs vibrarions au même inftant, les finiffent auft dans un même tems; on pourroir même unir ces deux pendules en fuppofant un corps extrèmement pefant, fufpendu par une très longue corde, \& un petit corps attaché au grand par un petit fil, on pourra toujours obtenir que le petit pendule attaché au grand commence la premiere de fes vibrations, \& finiffe la derniere, aux mêmes inftans que le grand pen. dule commence \& finit une feule vibration; mais fera-t-on fondé pour cela d'appeller ces vibrations ifochrones?

XII. Voyons encore fi toutes les nouvelles courbes trouvées par M. Euler, font comprifes dans notre remarque. Pour cet effet il faudra donner une équation pour toutes les courbes Tayloriennes, dont les cinq premieres figures font autant d'exemples. Je me fervirai des dénominations de M. Euler. Soit donc la longueur de la cor. de $\mathrm{AB}=a ; \pi=$ à la demi-circonference du cercle dont le rayon eft exprimé par l'unité, la plus grande appliquéc au milieu de chaque

ventre 


\section{$\quad 157$}

ventre pour la premiere figure $=a$, pour la feconde $=6$, pour la troifieme $=\gamma$, pour la quatrième $=\delta$; foit enfin $x$ une abfeiffe quelconque, \& y lappliquée pour cette abfcifle, on aura fuivant M. Taylor,

$$
\begin{aligned}
& \text { pour la r. fig. } y=a \text { fin } \frac{\pi x}{a} \\
& \text { pour la 2. fig. } y=6 \text { fin } \frac{2 \pi x}{a} \\
& \text { pour la 3. fig. } y=\gamma \text { fin } \frac{3 \pi x}{a} \\
& \text { pour la 4. fig. } y=\delta \text { fin } \frac{4 \pi x}{a} \text { \&c. }
\end{aligned}
$$

En combinant done toutes ces courbes à l'imitation de la figure fixième, pour laquelle nous n'avons combiné que les deux premieres figures, nous aurons généralement pour la même abfeifle $x$ cette équation

$y=\alpha$ fin $\frac{\pi x}{a}+6$ fin $\frac{2 \pi x}{a}+\gamma \operatorname{lin} \frac{3 \pi x}{a}+\delta \operatorname{lin} \frac{4 \pi x}{a}+\& \mathrm{c}$. dans laquelle les quantités $a, 6, \gamma, \delta, \& c$ font arbitraires affirma. tives ou négatives.

XIII. Voilà donc cette infinité de courbes trouvées fans aucun calcul, \& notre équarion eft la mêrne que celle de M. Euter ; voyez les Mémoires de l'Académic pour l'Annéé r748. page 85 . Il eft vrai que M. Eulev, ne traite pas cette multitude infiniment infinie de générale, \& qu'il ne la donne su f. 30 . que comme des cas particuliers; mais c'elt fur quoi je ne fuis pas encore aftez éclairci : s'il y a encore d'autres courbes, je ne comprens pas dans quel fens on peut les admettre.

XIV. Si dans notre équation on fuppofe les coëfficiens des termes alternes, favoir $\epsilon, \delta, \& c .=0$, i arrivera alors non feulement, que 


\section{踏 158}

toures les efpeces de vibrations finiffent au même inftant que finit la vi. bration fondamentale, mais encore que tous les points de la corde fe rangent au même inftant dans la pofition de la ligne droire $\mathrm{AB}$; cela n'eft qu'un corollaire de notre 6 . IO. Dans cette fuppofition la courbe obrient un diametre qui paffe par le milieu de la corde $A B$, \& qui partage la courbe en deux branches parfaitement égales \& femblables. Ce théoreme elt donc encore le même que celui de M. Eviler du §̧. 28.

XV. Si après toures nos remarques on vouloit encore confondre les vibrations compofées avec les vibrations fimples expofées par M. Trylor, je ne m'y oppoferai pas; mon intention n'a été principalement que d'expofer ce que les nouvelles vibrations de Mrs. d'Aiembert \& Euler ont de phyfique. Si au contraire on trouve que ces nouvelles vibrations ne fauroient être prifes pour des vibrations fimples, qui feules faifoient lobjet de M. Taylor, \& qu'étant décompolées en vibrations fimples \& uniformes, chaque efpece fe fait fimplement fuivant la loi de Taylor, ces nouvelles courbes ne feront que confirmer la théoric de M. Taylor, quand il exclud toutes les autres courbes, \& qu'il n'admet que fa trochoïde prolongée. Mais je n'en admirerai pas moins la profonde fagaciré avee laquelle nos deux illuftres Géometres ont fu dérerminer analytiquement ces nouvelles courbes. Au refte je crois que quelque courbure initiale qu'on donne à la corde, ellene man. quera pas de faire fes vibrations presque auffitôt fuivant la fimple uniformité des mouvemens ifochrones, \& conformèment à la nature de la trochoïde prolongée expofée par M. Tirylar, quoique Mr. Euler ne paroiffe pas de ce fentiment au §.27. Voici comment je conçois la chofe. L'expérience \& la raifon nous apprennent, que de deux cor. des également groffes \& également tenduës, la plus longue conferve plus longtems fes vibrations que la plus petite; dans les petites cordes pincées le fon ne dure qu'un inftant. Ainfi toutes les vibrations partielles, mêlées avec la vibration totale \& fondamentale, finiront bien vitre, pendant que la fondamentale dure encore très fenfiblement; \& alors la

corde 


\section{\& $159 \quad 8$}

corde ne formera plus que la trochoïde prolongée de M. Taylor. C'eft auffi ce que nous voyons toujours arriver dans les cordes pincées d'un clavecin, dans lesquelles on reconnoit affez par les yeux ladite courbe uniforme, qui ne forme qu'un feul ventre.

XVI. On peut encore remarquer fur cette matiere, qu'il n'y a que les cordes uniformement ćpaiffes, qui foient fufceptibles des propriérés que nous venons d'expofer; la raifon en eft, que lorfque les cordes inégalement épaiffes fe replient fuivant les figures 2.3.4.5. \&c. les vibrations n'en deviennent pas précifement $2 \cdot 3 \cdot 4 \cdot 5$. \&c. fois plus rapides qu'elles font par rapport à la première figure, \& qu'ainfi dans le mêlange de ces vibrations elles ne finiflent jamais dans un même inftant, quoiqu'elles ayent toutes commencé dans un même inftant. Il n'y a done à mon avis qu'une feule courbe pour les cordes inégalement épaiffes, pour un nombre d'interfections donné, qui fatisfaffe à notre problème ; \& fi cela eft, pourquoi y en auroit-il une infinité pour les cordes uniformement épaifles? Enfin, qu'on choififle le mà lange le plus fimple, en confidérant l'équation $y=\alpha$ fin $\frac{\pi x}{a}+6$ fin $\underline{2 \pi x}$, dans laquelle les coêfficiens $\alpha \& \mathscr{G}^{\circ}$ peuvent être de petires lignes quelconques. Soit la longueur du pendule fimple ifochrone, avec les vibrations uniformes qui répondent à la kmple première figure $=l$; la longueur d'un pareil pendule pour la feconde figure $=\frac{\pi}{4} /$; ou fuivant $\mathrm{M}^{\mathrm{rs}}$, d'Alembert \& Euler, le pendule ifochrone pour la figure exprimée par l'équation $y=a$ fin $\frac{\pi x}{a}+6$ fin $\frac{2 \pi x}{a}$ a toujours pour longueur la quantité l; cependant cette même longueur eft manifeftement $=\frac{3}{4} l$ en faifant $\alpha=0$; il y auroit done là dedans une contradiction, fi ces Géometres ne prenoient le mot difochronifme dans un autre fens, qu’on ne lui attache ordinairement.

XVII. 
槕 160

XVII. De notre folution fynthetique du problème de Mrs. $\mathrm{d} ' A$. lemilert \& Etler, on voit auffi fans caleul la maniere de déterminer la courbe abfoluë de la corde à cheque inftant, de mème que la viteffe de chaque point: car, foit la courbe initiale exprimée par

$$
y=\alpha \sin \frac{\pi x}{a}+6 \sin \frac{2 \pi x}{a}+\gamma \operatorname{fin} \frac{3 \pi x}{a}+\delta \sin \frac{4 \pi x}{a}+\& \mathrm{cc} .
$$

\& qu'il foir, par exemple, queftion de déterminer la courbe au moment que le milieu de la corde paffe par l'axe droit AB: il eft clair que la ourbe fera exprimée dans cet inftant par cette équation

$$
y=-6 \operatorname{fin} \frac{2 \pi x}{a}+\delta \text { fin } \frac{4 \pi x}{a}-\& c .
$$

dans laquelle les fignes font alternativement négatifs \& affirmatifs. Quant aux viteffes, il eft de même facile à les déterminer par la fimple compofition du mouvement, puifqu’on détermine dans laMéchanique le rapport des virefles de chaque point pour chaque figure à part.

XVIII. J'ai évité jufques ici les calculs, \& j'ai fondé tout mon raifonnement fur le principe confirmé par T'expérience (\$.6.) qu'il peut fe faire un mêlange de vibrations dans un feul \& même corps fonore, qui foient abfolument indépendantes les unes des autres. $A$ 'bien confidérer ce principe, il n'eft pas differant de celui de la compofition du mouvement ; cependant, pour le mettre dans tout fon jour, $\vec{\jmath}$ ai cru devoir ajouter ici les réfléxions méchaniques, \& les calculs que cette matiere demande.

XIX. Soit un corps A (fig.7.) tiré directement vers un point fixe B: fi l'on demande que le corps arrive au point $\mathrm{B}$ dans un tems donné, quelle que foit la diftance initiale $A B$, on fait que les gravitations du corps $\mathrm{A}$ vers le poimt fixe $\mathrm{B}$, doivent être dans chaque point proportioneltes gux diftances du corps depuis le point $\mathrm{B}$. On fait même, qu'il n'y a que cette feule loi de gravitation qui fatisfaffe à ta ques- 
85 $16 \mathrm{I} \quad$ है

tion; alors les demi-excurfions deviennent de part \& d'autre pasfai. tement égales. Voilà le vrai ifochronifme, \& le feul qu'il faille confidérer. Suppofons après cela que, pendant que le corps $A$ eft pouffé avec la dite loi vers le point $B$, les deux points $A \& B$ fouffrent dans chaque inftant une accéleration égale vers le point $\mathrm{C}$, de forte que le fyftème A B fouffre une gravitation commune vers $C$, qui foir encore proportionelle aux diftances $\mathrm{BC}$; par là on obtiendra un autre ifochronifme dans les excurfions du fyftème A B. Après cela on pourra de nouveau imaginer que les trois points $A, B, \& C_{5}$, fouffrent dans chaque inftant une accélération égale vers un quatrième point $\mathrm{D}$, \& que cette gravitation commune aux trois points $A, B, \& C$, foic toujours proportionelle aux diftances du point $\mathrm{C}$ au point $\mathrm{D}$. De cette façon on pourra multiplier à l'infini les gravitations du corps $\mathrm{A}$ vers differens points, \& ce corps A fouffrira par là un mellange de plufieurs efpeces de vibrarions, qu'il convient d'examiner.

XX. Quelle que foit la diftance initiale, le corps A employera toujours le même tems pour parvenir au point $B, \&$ pour faire une demi-vibration; aprc̀s quoi le corps $\mathrm{A}$ fera une autre demi-vibration, du côté oppofé toujours dans le mème tems; il reviendra encore au point $B$ après un troifième tems, \& àni de fuite: en un mot ce mouvement rélatif au point $B$ fera le même, que fi ce point demeuroit entiérement en repos. J'appellerai ces motvvemens réciproques, des vibrations ifochrones rélatives; \& cette diftinction des vibrations elt bien néceflaire, parce qu'en effet elles font bien différentes des vibrations abfolutis du même corps $A$. Suppolons que la pefanteur du corps $A$ vers le point $B$ foit Cgale à la pefanteur naturelle, en faifant fa diftance au point $B=a$; dans cette fuppofition ces premieres vibrations ifochrones rélatives feront ifochrones avec les vibrations abfolutis diun pendule fimple de la longucur $a$.

A près avoir confidúré ces premiercs vibrarions rélarives au point $\mathrm{B}$, nous aurons les mèmes réfléxions à faire fur les vibrations ifochro- 


\section{氶 162 疎}

nes du point $B$, rélatives au point $C ; \&$ ces vibrations exifteront en me me tems dans le corps $A$, fans troubler en aucune façon les premières vibrations de ce corps. Ces fecondes vibrations feront ifochrones avec celles d'un pendule fimple de la longueur $b$, en fuppofant que la pefanteur du point $B$, ou du corps $A$, vers le point $C$, devient égale à la pefanteur naturclle, lors qu'on fait $\mathrm{BC}=b$.

Si l'on veut ajouter à ces doubles vibrations du corps $\mathrm{A}$ une troifième efpece, il faudra fuppofer une troifième vibration commune au fyftème précedent $\mathrm{ABC}$ vers un point $\mathrm{D}$, qu'on fera fous de pareilles dénominations ifochrones avec celles d'un pendule fimple de la longueurc. De cette maniere on pourra aller auffi loin qu'on voudra, \& produire dans le corps $\mathbf{A}$ tant d'efpeces de vibrations ifochrones réla. tives qu'on fe fera propofé. Mais n'allons pas plus loin, \& fuppofons cette troifième vibration abfolué, en confidérant le point $\mathrm{D}$ comme fixe. De cette façon le feul \& méme corps $\mathrm{A}$ aura en même tems trois efpeces de vibrations indépendantes les unes des autres, dont les eentres de force font les points B, C, \& D, que nous allons confidérer de plus près.

XXI. Les tems desdites vibrations partielles feront en raifon fous-doublée des quantités arbitraires $a, b$, \& $c$; fi ces quantités font, par exemple, comme 4, 9, \& 36 , les tems des vibrations feront comme 2,3 , \& 6; ainfi, pendant que le point $\mathrm{C}$ fair une vibration entiere rélativement au point fixe $\mathrm{D}$, le point $\mathrm{B}$ en fera deux rélarivement au point $C$, \& le point $A$ en fera trois rélativement au point $B$ : d'où il eft clair, que tout le fyftème ne fera remis dans fon premier érat qu'au bout de fix vibrations rélatives au point $B$, ou de quatre vibrations ré. latives au point $\mathrm{C}$, ou de deux vibrations rélatives au point fixe $\mathrm{D}$. Mais, fuivant la maniere dont Mrs. d'Alembert \& Euler envilagent la chofe, tout ce mêlange de vibrations ne feroit jamais que deux vibrations fimples. Dans cet exemple il n'arrivera point, que les trois points $A, B, \& C$, fe réüniffent au point fixe D. Mais fil l'on fuppofe les trois

quanti- 


\begin{abstract}
25 $\quad 163$
quantités arbitraires $V a, V b, \& V_{c}$, ètre en raifon de $3,5, \& 15$, les tems des vibrations feront en metme raifon, \& les points $A, B, \& C$ fe trouveront réünis au point $\mathrm{D}$ au bout de chaque demi-vibration du point $\mathrm{C}$; de cette maniere les excurfions feront parfairement femblables \& égales de chaque còté du point fixe $\mathrm{D}$ : mais à mon avis le point A n'en doit pas moins être cenfé faire cing demi-vibrations avant que cela arrive, au lieu que dans l'autre fens il n'eft cenfé de faire qu'u. ne feule demi-vibration. Pour voir la nécellité d'envifager ces mouvemens réciproques conformément à notre théorie, il n'y a qu'à fuppofer les diftances arbitraires initiales BC, \& CD, extrèmement petites par rapport à $A B$; il n'y aura alors que les vibrations $A B$ qui foyent fenfibles, \& le corps A fera manifeftement cinq demi-vibrations avant la rúūnion parfaite des points $\mathrm{A}, \mathrm{B}, \mathrm{\&} \mathrm{C}$, au point D. Mais, fi on confidéroit les diftances $\mathrm{A} B, \& \mathrm{BC}$, comme extrèmement petires par rap. port à $C D$, il n'y aura que les vibrations $C D$ qui foyent fenfibles, \& le corps A ne pourroit plus être cenfé que d'avoir fait une feule demivibration. Il faut done éviter ici toure équivoque dans les mots de vibration \& difochronisme. Si les quantités $V a, \boldsymbol{V} b, \& \boldsymbol{V} c$, font incommenfurables, il ne pourra jarnais arriver, que le fyftème reprenne fon érar initial, ni que le corps $\mathbf{A}$ revienne au point dont il étoir parti, parce que les points $\mathrm{A}, \mathrm{B}, \& \mathrm{C}$, nauront jamais leurs plus longues élongations rúlatives dans un même inftant : cependant chaque efpece de vibration relative à part fe fera fuivant la loi des pendules fimples cycloïdiques. C'eft la fource de la raifon, qui empéche d'étendre la nouvelle théorie de Mrs. d'Alembert \& Euler à toutes fortes de cordes, quelque loi d'inégalité qu'il puiffe y avoir dans leur épaiffeur.
\end{abstract}

XXII. Dans l'application de ces réfléxions aux cordes vibrantes, il faudra fuppofer $b=\frac{t}{4} c, a=\frac{\pi}{b} c \&$ ce. de forte que les nombres des differentes efpeces de vibrations fimples foyent en raifon des nombres naturels $\mathrm{r}, 2,3$, \&c. en commençant par celle du point $\mathrm{C}$ rélati-

$$
\mathrm{X} 2
$$

ve 


\section{舫 $164 \quad \%$}

ve au point fixe $\mathrm{D}$, enfuite celle du point $\mathrm{B}$ rélative à fon centre de force mobile $\mathrm{C}$, \& puis celle du point $\mathrm{A}$ rélative au centre de force mobile $\mathrm{B}$.

XXIII. Suppofons à préfent ha diffance initiale $\mathrm{A} \mathrm{B}=二 \alpha$, $\mathrm{BC}=6, \mathrm{CD}=\gamma, \& \mathrm{c}$ \& \& qun'an bour d'un tems donné $t$ la diftan. ce $\mathrm{AB}$ foit devenuë $=x$, la diftanee $\mathrm{BC}=y$, la liftance $\mathrm{CD}=\approx$ \&ce; foit aulfi la viteffe rélative entre les points $\mathrm{A} \& \mathrm{~B}=u^{\prime}$, celle d'entre les points $\mathrm{B} \& \mathrm{C}={ }^{\prime \prime \prime}$, celle d'entre les points $\mathrm{C} \& \mathrm{D}={ }^{\prime \prime}{ }^{\prime \prime}$ \&ce. on aura

$$
\begin{aligned}
& d u^{\prime}=\frac{x}{a} d t \quad \& \quad u^{\prime}=\sqrt{a, a-x} \frac{x}{a}, \\
& d u^{\prime \prime}=\frac{y}{b} d t \quad \& \quad u^{\prime \prime}=v^{66-y y}{ }_{b}, \\
& d u^{\prime \prime \prime}=\frac{\varepsilon}{c} d t \quad \& \quad u^{\prime \prime \prime}=V \frac{\gamma \gamma-\Sigma s}{c}, \& c .
\end{aligned}
$$

Outre ces équations la Géometrie nous enfeigne encore des conftructions \& équations entre le tems $t \&$ les diftances $x, y, \approx, \& c$. Car, foit le finus total exprimé par l'unité, \& qu'on dénote par A. cofF un are de cercle, dont le rayon eft un, \& dont le cofinus eft une quantité quelconque $\mathrm{F}$, on pourra exprimer le tems $t$ par chacune des équations fuivantes

$$
\begin{aligned}
t & =V a \times \text { A. } \operatorname{col} \frac{x}{a} \\
t & =V b \times \text { A. } \operatorname{col} \frac{y}{6} \\
t & =V c \times \text { A. } \operatorname{col} \frac{\approx}{\gamma} \& c .
\end{aligned}
$$

Par le moyen de ces équations on pourra déterminer chacune des dis.

tances 


\section{अ 165 \&}

tances $x, y, z$, \&ce. par le même tems $t$ commun, en convertiflant les fignes; de cette façon on aura

$$
\begin{aligned}
& x=\alpha \operatorname{col} \text { A. } \frac{t}{\sqrt{a}} \\
& y=6 \operatorname{col} \text { A. } \frac{t}{\sqrt{b}} \\
& \approx=\gamma \operatorname{col} \mathrm{A} \cdot \frac{t}{\sqrt{c}} \& \mathrm{c} .
\end{aligned}
$$

Dans ces équations il faut entendre par $\operatorname{cof} A \frac{t}{\sqrt{a}}$ le cofinus d'un arc de cercle, qui eft égal à $\frac{t}{\sqrt{a}} \&$ dont le rayon eft égal à l'unité, \& ainfi des autres. Outre cela les quantités $V a, V b, V c$, \&c. marquent les tems, qu'un corps animé par la pefanteur naturelle employe pour tomber librement des hauteurs $\frac{1}{2} a, \frac{1}{2} b, \frac{1}{2} c, \& c$.

Nous avons done déterminé chacune des quantités $x, y, z$, \&c. par le même tems $t$, \& par conféquent la diftance abfoluë du corps au dernier point fixe, laquelle diftance eft $=x+y+z+\& c$. Nous avons pareillement déterminé pour chaque inftant les viteffes rélatives $w^{\prime}, w^{\prime \prime}, w^{\prime \prime \prime}, \& c$. \& la viteffe abfoluẽ du point $\mathrm{A}$ exprimée par $u^{\prime}+w^{\prime \prime}+t^{\prime \prime \prime}+\& c$. de même que les éémens de toutes ces viteffes.

XXIV. On pourra, au lieu des tems exprimés par $V_{a}, V b, V c$, \&c. confidérer les tems qui répondent à une vibration fimple rélative du point A par rapport au point B; \& puis du point B par rapport au point $\mathrm{C}$, enfuite du point $\mathrm{C}$ par rapport au point $\mathrm{D}$. $\mathrm{Si}$ nous défignons donc ces tems par $\theta^{\prime \prime}, \theta^{\prime \prime}, \theta^{\prime \prime \prime}$ \& $\&$. \& que $\pi$ foit la demi-circon. férence d'un cercle qui a l'unité pour rayon, il faudra fuppofer dans 
166

les équations du precédent article $V_{a}=\frac{\theta^{\prime}}{\pi}, V_{b}=\frac{\theta^{\prime \prime \prime}}{\pi}, V_{c}=\frac{\theta^{\prime \prime \prime}}{\pi}$, après lesquelles fubftitutions toutes les quantités deviennent manifeftement homogenes.

Soit, par exemple, $\theta^{\prime}=\mathbf{x}^{\prime \prime}, \theta^{\prime \prime}={ }^{\prime \prime}, \theta^{\prime \prime}=3^{\prime \prime}, \&$ qu'il foit queftion de déterminer toutes les circonftances du mouvement en faifant $t=$ un quart de feconde; je dis qu'on aura $x=\alpha \operatorname{col} \mathrm{A} \frac{\pi t}{\theta^{\prime}}=\alpha \operatorname{col} \mathrm{A} \frac{1}{4} \pi=\alpha V_{\frac{1}{3}} ; y=6 \operatorname{col} \mathrm{A} \frac{\pi t}{\theta^{\prime \prime}}$ $=6 \operatorname{col} \mathrm{A} \frac{\mathrm{t}}{8} \pi=6 \operatorname{col} 22^{\circ} 3^{\prime}, \& s=\gamma \operatorname{col} \mathrm{A} \frac{\pi}{12}=\gamma \operatorname{col} 15^{\circ}$; donc $x+y+z=0,70710 a+0,923876+0,96992 \gamma$, laquelle quantité marque la diftance du corps $\mathrm{A}$ au point $\mathrm{D}$ après un quart de feconde de tems. On aura enfuite $t^{\prime}=v \frac{\alpha a-x x}{a}$ $=\frac{a}{\sqrt{a}}$ fin $\mathrm{A} \frac{1}{4} \pi ; \quad$ st $=v \frac{66-y y}{b}=\frac{6}{\sqrt{b}}$ fin $\mathrm{A}+\pi=$ $\frac{6}{2 \sqrt{ } a}$ fin $A t \pi, \& w^{\prime \prime \prime}=\frac{\gamma}{3 \sqrt{ } a}$ fin $A_{r^{\prime} z} \pi$. De là il réfulte pour notre exemple la vitefle abfoluẽ $u^{\prime}+u^{\prime \prime}+u^{\prime \prime \prime}=$

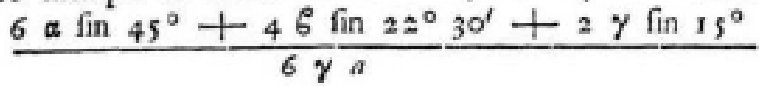

XXV. Mais, fans nous arrêter à cette forte de déterminations, qui n'ont pas trop de rapport avec ce que je me fuis propofé de principal, examinons plutôt fous quelles circonftances la viteffe ablolué $w^{\prime}+w^{\prime \prime}+w^{\prime \prime \prime}$ peut devenir $=0$, en nous bornant aux trois efpeces de vibrations fimples mélées enfemble. Il faudra pour cet ef: fer, en vertu du §. 23. faire $V \frac{\alpha \alpha-x x}{a}+\gamma \frac{66-y y}{b}+\gamma \frac{\gamma y-3 s}{c}=0$

fubfti- 


\section{क 167 क}

fubftituons pour $x, y, \&$ a leur valeur en $t, \&$ nous aurons $\frac{a}{\sqrt{a}}$ fin $A \frac{t}{\sqrt{a}}+\frac{6}{\sqrt{b}}$ fin $A \frac{t}{\sqrt{b}}+\frac{\gamma}{\sqrt{c}}$ fin $\mathrm{A} \frac{t}{\sqrt{c}}=0$. Je me propofe de déterminer la valeur de $\boldsymbol{t}$, qui fatisfaffe à cetre équation dans quelque exemple particulier. Nous fuppoferons done $f^{\prime \prime \prime}$ ou $\pi V_{c}=2 \theta^{\prime \prime}$ ou $2 \pi V b=3^{A^{\prime}}$ ou $3 \pi V a$, c'eft à dire nous fuppoferons, que, pendant que le point $C$ fait une vibration pour le point fixe 1 , le point $B$ en falfe deux pour le point $C, \&$ le point $\Lambda$ en fiffe trois pour le point $\mathrm{B}$, laquelle fuppofition demande $V c=2 \sqrt{ } b={ }_{3} V_{a}$. Là deffus notre équation donnera

$$
3 a \text { fin } A \frac{3 t}{V c}+26 \text { fin } \Lambda \frac{2 t}{V c}+\gamma \text { in } A \frac{t}{V c}=0 .
$$

Soit à préfent fin $\mathrm{A} \frac{t}{\sqrt{c}}=q, \&$ on aura fin $\mathrm{A} \frac{2 t}{\sqrt{c}}$, c'cft à dire le finus de larc double $=2 q V(t-q q) \&$ fin $A \frac{3 t}{V_{c}}=3 q-4 q^{\prime}$, \& là deffus nous aurons

$$
\begin{gathered}
3 a\left(3 q-4 q^{3}\right)+26 \times 2 q V(x-q q)+\gamma q=0, \\
\text { ou bien } \\
2 a-12 \alpha q q+46 v(1-q q)+\gamma=0 .
\end{gathered}
$$

Par la premiere des ces équations on voit dejà, qu'on peut faire $1=\sin A \frac{t}{V_{c}}=0$, ce qui donne $2=\gamma \operatorname{col} A \frac{t}{V_{\epsilon}}= \pm \gamma$; $y=6 \operatorname{cof} \mathrm{A} \frac{t}{\sqrt{b}}=6 \operatorname{cof} \mathrm{A} \frac{2 t}{\sqrt{c}}=\mathbf{6}, \& x= \pm \alpha, \&$ par conféquent $x+y+z= \pm a+6 \pm \gamma$. C'eft la le cas qui s'offre de foi . même; mais il y en a encore d'autres indiqués par la feconde équarion, qui peuvent être très réels. Pour abréger le calcul, nous nous borne.

rons 


\section{爱 168 承}

rons aux cas qui font $9 \alpha+\gamma=46$; \& dans ces cas on trouve le fin $\mathrm{A} \frac{t}{V_{c}}$ ou bien

$$
q= \pm \frac{V(6 a b-6 b)}{3 \alpha} ;
$$

\& enfuite le $\operatorname{cof} \mathrm{A} \frac{t}{V c}$ ou bien

$$
V(\mathrm{r}-9 q)= \pm \frac{V(9 \alpha \alpha-6 \alpha b+66)}{3 \alpha}= \pm\left(1-\frac{6}{3 \alpha}\right) \text {. }
$$

De là on obticnt $\approx= \pm \gamma\left(\mathrm{t}-\frac{6}{3 \alpha}\right)$,

cettc valeur de $\approx$ marque déjà la pofition du point $\mathrm{C}$; on trouvera enfuite la valeur de $y$, \& enfin celle de $x, \&$ par là on aura déterminé les pofitions des trois points $\mathrm{C}, \mathrm{B}$, \& $\mathrm{A}$.

On voit done que notre queftion eft le plus fouvent fufeeptible de plufieurs folutions très réelles, quoiqu'elles ne le foyent pas toujours: car dans le cas préfent il faut que la quantité $\frac{6}{3 \alpha}$ foit tovjours un nom. bre affirmatif, \& que 6 foit plus perit que $6 a$.

Defcendons ici à un exemple tout particulier: fuppofons $6=4 a$ $\&$ par confequent $\gamma=7 \alpha$. Dans ce cas il faut prendre $V(1-q q)$ négativement, \& faire $V(x-q q)=-\frac{t}{5}$, ce qui donne l'arc $\frac{t}{V_{c}}$ de $109^{d}, 28^{\prime}$, dont le cofinus eft $=-\frac{t}{5}$; l'are $\frac{2 t}{V c}$ de $218^{d}, 56^{\prime}$, dont le cofinus eft $=-0,77787 ; \&$ l'arc $\frac{3 t}{V_{c}}$ de $328^{\alpha}, 24^{\prime}$, dont le cofinus eft $=0,83172$. Ces valeurs donnent $s=-\frac{1}{3} \gamma=-\frac{7}{3} \alpha$; 
\& $169 \quad 8$

$y=-0,777876=-3,11148 \alpha ; \& x=0,85172 \alpha, \&$ par conféquent $x+y+\approx=-4,59309 \alpha$. Ainfi la diftance initiale du corps A depuis le point D étant exprimíe par I 2, il y aura encore deux pofitions du corps $\mathrm{A}$, dans lesquelles fa viteffe abfoluë foit nulle, \& fes diftances au point D feront alors $-4 \&-4$, 59309 .

XXVI. Voici à prefent la narure du mouvement ablolu du corps A par rapport au point fixe $\mathrm{D}$; la figure 8 marque les points capitaux avec la jufte proportion des diftances. Je dis done que le corps partant du point $\mathrm{A}$ fera d'abord accéléré \& enfuite retardé, après quoi il fera arrêté pour un moment au point B; il rebrouftera de là jusques au point $\mathrm{C}$, où il repofera encore un inftzht; du point $\mathrm{C}$ il retournera vers le point B en s'y arrêtant de nouveau pendant un inftant, \& du point $B$ enfin il retournera au point $\mathbf{A}$ dont il étoit parti, où fa viteffe fera encore nulle, \& alors tout fera réduit à fon premier érat. Outre cela le tems employé pour faire la premiere vibration A B eft exprimé par l'are rogd $^{d}, 28^{\prime}$, le tems pour faire la feconde petite vibration BC par l'are $70^{d} \cdot 3_{2}^{\prime}$; ce fecond tems fera encore employé pour faire la troifième vibration $C B, \&$ le premier tems pour faire la quatrième vibra. tion B A. Si on exprime donc tout le tems, que le corps employe avant que de fe remetrre en fonpremier état, par 2 1600, la premiere vibration employera 6568 de même que la quatrième, pendant que chacune des deux moyennes employera 4232 . Comme dans cet exemple la feconde \& la troifième excurfion font très petites, le mouvement du corps paroitra comme presque en repos pendant prefque la moitić du tems entier. Mais on auroit pu choifir d'autres exemples, où les excurfions partielles feroient devenuës comme égales \& comme ifochrones, $\&$ alors c'eft une queftion, fi le corps doit être cenfé avoir fait quatre vibrations, ou deux; dans le fens de Mrs, d'Alembert \& Euler, il faudroit toujours dire, qu'il n'en auroit fait que deux, quoiqu'on put lui faire faire cent allées \& venuěs toutes prefque égales, \& prefque ifochrones, \& dont les mouvemens fe feroient prefque entierement felon les loix des mouvemens ifochrones fimples \& ordinaires.

Nim, defAsed, Tom. IX.

XXVII. 




XXVII. Ceque je viens de dire du mouvement du corps $\mathrm{A}$, ar. rive parfaitement à chaque point dans les nouvelles vibrations des cordes: je me contenterai, pour faire voir cette entiere conformité, d'expliquer le mellange des deux premieres efpeces de vibrations les plus fimples, exprimées par les deux premieres figures. J'ai repréfentéce mêlange par la figure fixième, dont j’ai donné la conftruction au $\$ .8$. Soit dans cette courbe abfoluë $\mathrm{A}$ p $a$ \& $\mathrm{B}$ la plus grande appliquée rélative $p m=\rho$, la plus grande appligucée a $r$ de la courbe idénle A $m a n \mathrm{~B}=\sigma$; \& foit encore la longueur de la corde entiere $\mathrm{AB}=a$; qu'on prenne une abfciffe quelconque $\mathrm{A} b=x$ avec fon appliquée $\boldsymbol{b} d$; là -deffus on aura en vertu de la Théorie de M. Taylar $c d=\rho$ fin $\frac{2 \pi x}{a} \& b c=\sigma$ fin $\frac{\pi x}{a}$, \& par conféquent l'appliquée $b d=\rho$ fin $\frac{2 \pi x}{a}+\sigma$ fin $\frac{\pi x}{a}$. Soit $b d=y$, on aura, en fuppofant $d x$ conftante, $d d y=\left(\frac{4 \pi \pi}{a} \frac{\pi}{a} \rho \sin \frac{2 \pi x}{a}+\frac{\pi \pi}{a a} \sigma\right.$ in $\left.\frac{\pi x}{a}\right) d x^{2}$; or, nommantPle poids qui tend la corde, \& $p$ le poids de la corde tendue \& uniforme, on fait que la force accélératrice abfoluë du point $d$ eft exprimée par $\frac{\mathrm{P}}{p} \cdot \frac{a d d y}{d x^{2}}$; cette force accélératrice fera donc ici $\frac{\mathrm{P}}{p} a\left(\frac{4 \pi \pi}{a a} \rho\right.$ in $\frac{2 \pi x}{a}+\frac{\pi \pi}{a a} \sigma$ fin $\left.\frac{\pi x}{a}\right)$ ou bien $=$ $\frac{4 \mathrm{P}}{p} \cdot \frac{\pi \pi}{a} \rho$ fin $\frac{2 \pi}{a}-\frac{x}{p}+\frac{\mathrm{P}}{p} \cdot \frac{\pi \pi \sigma}{a}$ fin $\frac{\pi x}{a}$, ce qui fait la force accélératrice entiere du point $d=\frac{4 \mathrm{P}}{p} \cdot \frac{\pi \pi}{a} \cdot c d+\frac{\mathrm{P}}{p} \cdot \frac{\pi \pi}{a} \cdot b c$. Nous voyons done, que la force accélératrice entiere eft compof́ée de deux parties, dont la premiere eft proportionelle à la diftance $d c$, \& qui fait faire au point $d$ des vibrations ifochrones rélatives au point $c$, qui 
क $171 \quad$ *

fair ici un centre de force mobile: la feconde partie de la force accéle ratrice du point $d \mathrm{eft}==\frac{\mathrm{P}}{p} \cdot \frac{\pi \pi}{a}, b c:$ or, par la nature de la courbe A $c a n \mathrm{~B}$, le point $c$ eft animé vers le point immobile $b$ avec la mème force accélératrice $\frac{\mathrm{P}}{p} \cdot \frac{\pi \pi}{a}, b c$; ainfi la feconde force produit conftamment la même accélération aux points $d \& c$, \& ne fera que transporter la petite diftance $d c$ fans la changer aucunement. Nous fommes donc entierement dans le même cas que celui des $\$ \$$. 19 . \& 20. Pour obrenir une entiere identité il faudra fuppofer dans la fep. tième figure $\mathrm{C} \mathrm{D}=o, \mathrm{AB}=d c \& \mathrm{BC}=c b$, ou bien $\alpha=\rho$ fin $\frac{2 \pi}{a}, \varepsilon=\sigma$ fin $\frac{\pi x}{a} \& \gamma=0, \&$ dans ces expreffions la quantité $x$ eft conftantepar rapport au mouvement du même point $d$. Ainfi le mouvement de chaque point dans la courbe A da q B, fera par rapport à la courbe idéale $\mathrm{A} \operatorname{can} \mathrm{B}$, le même que le mouvement abfolu de la feconde figure, \& le mouvement abfolu de la courbe idéalo A c a $n$ B fera le mêne que celui de la premiere figure; ainfi la vibra. tion fuivant la figure A $d a$ q $\mathrm{B}$ n'eft abfolument qu'un mêlange des vibrations fuivant la premiere \& la feconde figure combinées enfemble, dont chsque efpece fe fait indépendamment l'une de l'autre. Ce mou. vement abfolu du point $d$ renferme réellement deux mouvemens périodiques, l'un par rapport au point $c$, \& l'autre par rapport au point $b$; le nombre des premiers retours périodiques fera toujours double de celui des feconds. L'efprit-s'apperçoir de l'une \& de l'autre efpece de ces retours périodiques, \& remarque par là un double fon, dont l'un eft l'octave de l'aurre. Comme les petites quantités $p m$ \& a r défignées par $\rho \& \sigma$ peuvent avoir un rapport quelconque, on ne remarquera que la premiere efpece de mouvemens périodiques en faifant $p m$ beaucoup plus grande que $a r$, pendant qu'on ne fentira que la feconde efpece en faifant $a r$ beaucoup plùs grande que $p m$; dans le premier 


\section{象 172 数}

cas le tems d'une vibration eft manifeftement de la moitié plus perix que dans le fecond, pendant que, felon la maniere d'envifager ces vibrations de Mrr. d'Alembert \& Euler, le tems de chaque période eft toujours le mêtme, quelque rapport qu'il $\mathrm{y}$ ait entre $\rho \& \sigma$, ce qui ne me paroit pas conforme au principe de continuité. Car fi le tems d'une vibration eft felon eux toujours $t$, quelque rapport qu'il y aitentre $\rho \& \sigma$, pourquoi devient-il manifeftement $\frac{1}{2} t$ en faifant $\sigma=0$ ? Pour répondre à cette difficulté, il me femble qu'il faut néceflairement dire, qu'il fe fait en même tems une double efpece de vibrations dans la corde, \& que le mêlange de ces deux efpeces forme ce, que ces Géome. tres appellent vibrarions fimples; que la feconde efpece diminuë à mefure qu'on diminus la quantité $\sigma, \&$ qu'elle devient nulle enfaifant $\sigma=0$; $\&$ alors il n'y aura aucune difcontinuité.

XXVIII. J'efpère que ce que je viens de dire dans ce Mémoire pourra fervir à répandre plus de jour fur la narure des nouvelles vibrations des cordes, trouvées avec tant de fagacité par $M n$. d'Alemblert \& Evler; \& c'étoit hà tout mon but. Si la méthode dont ils fe font fervis pour réloudre leurs problèmes eft beaucoup plus difficile que la mienne, je n'en admire que d'avantage la fupériorité de leur génie. Quant à la queftion, fi les nouvelles vibrations font réellement des vibrations fimples \& fynchrones pour tous les points, ou fi elles ne font pas plutôt un mêlange de plufieurs différentes vibrations coēxiftanteg dans une měme corde, \& toutes de différente durée, je n'en ai parlé que pour mieux expliquer la nature de ces vibrations, étant bien éloigné de faire une quérelle à d'auffi grands hommes fur la fignification de certains termes.

Je traiterai dans un fecond Mémoire quelques nouveaux problemes, dont la folution découle affez facilement de nos principes, \& qui en même tems mettront cette matiere dans un nouveau jour. 


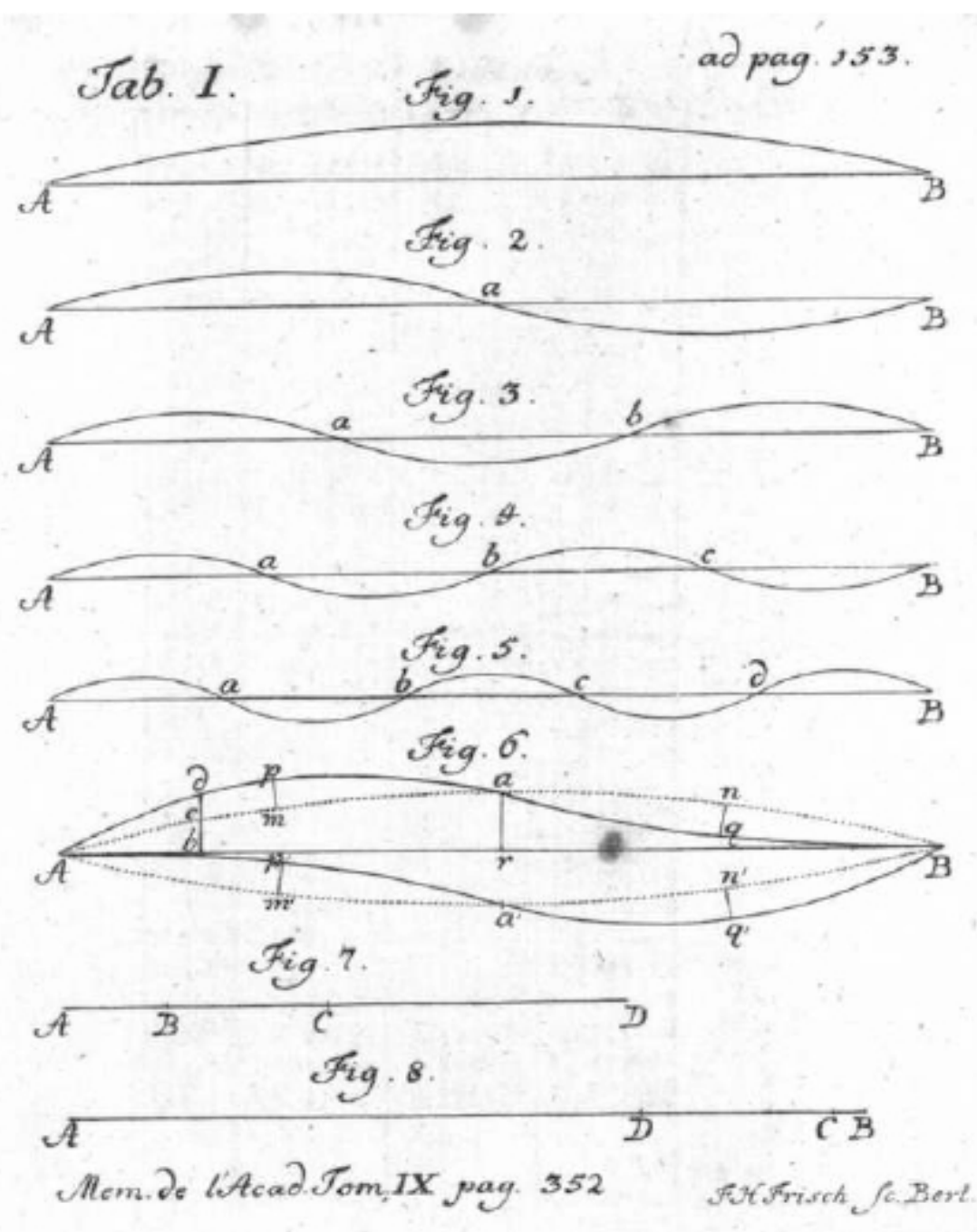

